Supporting Information for:

\title{
Poly-p-phenylene Phosphine/Polyaniline Alternating Copolymers: Electronic Delocalization Through Phosphorus
}

\author{
Zhou Jin and Brett L. Lucht \\ Department of Chemistry \\ University of Rhode Island \\ Kingston, RI 02881
}

Experimental procedures for the preparation of PPPP-PANI co-polymers and polymer model compounds. UV-visible spectra of the chemical oxidation of 9a, 9b, 10a, 10b, 11a, 11b, 12a, 12b, 13a, and 13b and cyclic voltammographs of $6 a, 6 b, 8 a, 8 b, 9 a, 9 b, 10 a, 10 b, 11 a, 11 b$, 12a and 12b. 


\section{Experimental}

\section{General Comments}

Organometallic reagents were stored and handled under nitrogen atmosphere in a glovebox or using Schlenk techniques. Dry, oxygen free solvents were employed throughout. Solvents were purchased from Aldrich Chemical Co. anhydrous and stored under nitrogen. All NMR spectra were acquired using a JEOL GSX $400 \mathrm{MHz}$ spectrometer. Infrared spectra were recorded on a Thermo Nicolet IR 300 FT-IR spectrometer. Elemental analyses were performed by Galbraith Laboratories, Inc. Elemental analyses of polymers $6 \mathbf{a}, \mathbf{6 b}, \mathbf{8 a}, \mathbf{8 b}, 9 \mathbf{a}$, and 9b were conducted multiple times and provided highly variable results and are not reported. Molecular weights of the polymers were determined by gel permeation chromatography (GPC; Agilent Technology series 1100 HPLC; Detector: Differential Refractometer G1362A; Pump: Isocratic 1310A; Software: Chemstation; Column: Plgel 10³ A) with THF as eluting solvent and polystyrene standards. Electrochemical measurements were carried out on an EG\&G instruments 263 A potentiostat/galvanostat using a standard cell, consisting of a solid $\mathrm{Pt}$ working electrode and $\mathrm{Ag} / \mathrm{AgCl}$ reference electrode $\mathrm{Pt}$ wire counter electrode. Scan rate is $50 \mathrm{mV} / \mathrm{s}$. The supporting electrolyte was tetrabutylammonium hexafluorophosphate in dry degassed methylene chloride. Ferrocene was used as an external standard. UV-Visible-NIR spectra were recorded on a PE Lambda 900 UV-Vis-NIR spectrometer.

$\boldsymbol{N}$ - $\boldsymbol{p}$-iodophenyl-p-anisidine. A $250 \mathrm{~mL}$ Schlenk tube was charged with $12.3 \mathrm{~g}$ (0.100 mol) of anisidine, $99 \mathrm{~g}$ (0.30 mol) of 1,4-diiodobenzene, $1.5 \mathrm{~g}$ (2.05 mmol) of $\mathrm{Pd}(\mathrm{DPPF}) \mathrm{Cl}_{2}$, and 32 $\mathrm{g}$ of $t$-BuONa in a dry box. $100 \mathrm{~mL}$ of toluene was added to the reaction mixture via cannula followed by heating at $70{ }^{\circ} \mathrm{C}$ for 5 days. The reaction mixture changed to a brick-red color and became homogenous after the first 30 min of heating. As the reaction continued, significant precipitate was formed. The reaction was stopped, and $150 \mathrm{~mL}$ of water was added to the reaction mixture followed by extraction with chloroform $(3 \times 50 \mathrm{~mL})$. The 
organic solvent was removed by evaporation to yield the crude product, which was purified by sequential column chromatography on silica gel in hexane followed by chloroform to yield 25 g (0.077 mol, $77 \%)$ of $N$-p-iodophenyl aniline as a white crystalline solid. ${ }^{1} \mathrm{H}$ NMR (400 $\left.\mathrm{MHz}, \mathrm{CDCl}_{3}\right): \delta 3.80\left(\mathrm{~s}, 3 \mathrm{H}, \mathrm{OCH}_{3}\right), 5.21(\mathrm{br}, 1 \mathrm{H}, \mathrm{NH}), 6.65\left(\mathrm{~d}, J=8.8 \mathrm{~Hz}, 2 \mathrm{H}, \mathrm{C}_{6} \mathrm{H}_{4}\right), 6.86(\mathrm{~d}$, $\left.J=8.8 \mathrm{~Hz}, 2 \mathrm{H}, \mathrm{C}_{6} \mathrm{H}_{4}\right), 7.04\left(\mathrm{~d}, J=8.4 \mathrm{~Hz}, 2 \mathrm{H} \mathrm{C}_{6} \mathrm{H}_{4}\right), 7.44\left(\mathrm{~d}, J=8.4 \mathrm{~Hz}, \mathrm{C}_{6} \mathrm{H}_{4}\right) ; \delta^{13} \mathrm{C}(100$ $\left.\mathrm{MHz}, \mathrm{CDCl}_{3}\right) 55.51$ (s, CH3), $80.23\left(\mathrm{~s}, \mathrm{C}_{6} \mathrm{H}_{4}\right), 114.83\left(\mathrm{~s}, \mathrm{C}_{6} \mathrm{H}_{4}\right), 117.45\left(\mathrm{~s}, \mathrm{C}_{6} \mathrm{H}_{4}\right), 122.86$ (s, $\left.\mathrm{C}_{6} \mathrm{H}_{4}\right), 134.86\left(\mathrm{~s}, \mathrm{C}_{6} \mathrm{H}_{4}\right), 137.88\left(\mathrm{~s}, \mathrm{C}_{6} \mathrm{H}_{4}\right), 145.18\left(\mathrm{~s}, \mathrm{C}_{6} \mathrm{H}_{4}\right), 155.81\left(\mathrm{~s}, \mathrm{C}_{6} \mathrm{H}_{4}\right)$; IR (ATR cm $\left.{ }^{-1}\right)$ $3420,3010,2959,2360,2341,1590,1510,1489,1457,1318,1298,1285,1243,1179,1109$, 1063, 1029,1000, 815,769. Mp $118{ }^{\circ} \mathrm{C}$ to $119.5{ }^{\circ} \mathrm{C}$. Anal. Calcd for $\mathrm{C}_{13} \mathrm{H}_{12} \mathrm{INO}: \mathrm{C}, 48.02 ; \mathrm{H}$, 3.72. Found: C, 47.96: H, 3.89.

(1) 1,4-bis(N-4'-n-butylphenylamino)benzene. A $250 \mathrm{~mL}$ Schlenk tube was charged with $11.8 \mathrm{~g}$ (0.05 mol) of 1,4-dibromobenzene, $22.4 \mathrm{~g}$ ( $0.15 \mathrm{~mol})$ of 4-butyl-aniline, $0.37 \mathrm{~g}(0.50$ mmol) of $\mathrm{Pd}(\mathrm{DPPF}) \mathrm{Cl}_{2}$, and $10.0 \mathrm{~g}(0.104 \mathrm{~mol})$ of $t-\mathrm{BuONa}$ in a dry box. $50 \mathrm{~mL}$ of toluene was added via cannula under nitrogen and the reaction mixture was allowed to stir at $110{ }^{\circ} \mathrm{C}$ for 2 days. The reaction mixture became red after about $15 \mathrm{~min}$ of heating, followed by the formation of precipitate. The reaction was quenched by the addition of water $(150 \mathrm{~mL})$. The reaction mixture was extracted with chloroform $(3 \times 50 \mathrm{~mL})$. The organic solvent was removed by evaporation to leave a dark tar. The tar was washed with hexane to remove residual starting materials, then dissolved in ethyl acetate and purified by column chromatography (silica gel in ethyl acetate) to yield light yellow crystals of $\mathbf{1}(12 \mathrm{~g}, 65 \%) . \quad{ }^{1} \mathrm{H}$ NMR (400 MHz, $\mathrm{CDCl}_{3}$ ): $\delta 0.74$ (t, $J=7.32,6 \mathrm{H} \mathrm{CH}_{3}$ ), 1.15 (mult, 4H, $\mathrm{CH}_{2}$ ), 1.37 (mult, 4H, $\left.\mathrm{CH}_{2}\right), 2.32\left(\mathrm{t}, J=7.72,4 \mathrm{H}, \mathrm{CH}_{2}\right), 4.75(\mathrm{~s}, 2 \mathrm{H}, \mathrm{NH}), 6.65\left(\mathrm{~d}, J=8.0,4 \mathrm{H}, \mathrm{C}_{6} \mathrm{H}_{4}\right), 6.66(\mathrm{~s}, 4 \mathrm{H}$, $\left.\mathrm{C}_{6} \mathrm{H}_{4}\right), 6.81\left(\mathrm{~d}, J=8.0,4 \mathrm{H}, \mathrm{C}_{6} \mathrm{H}_{4}\right) ; \delta^{13} \mathrm{C}\left(100 \mathrm{MHz}, \mathrm{CDCl}_{3}\right): 13.71\left(\mathrm{~s}, \mathrm{CH}_{3}\right), 22.22\left(\mathrm{~s}, \mathrm{CH}_{2}\right)$, $33.85\left(\mathrm{~s}, \mathrm{CH}_{2}\right), 34.85\left(\mathrm{~s}, \mathrm{CH}_{2}\right), 116.80\left(\mathrm{~s}, \mathrm{C}_{6} \mathrm{H}_{4}\right), 120.05\left(\mathrm{~s}, \mathrm{C}_{6} \mathrm{H}_{4}\right), 128.92\left(\mathrm{~s}, \mathrm{C}_{6} \mathrm{H}_{4}\right), 133.97(\mathrm{~s}$, $\left.\mathrm{C}_{6} \mathrm{H}_{4}\right), 137.39\left(\mathrm{~s}, \mathrm{C}_{6} \mathrm{H}_{4}\right), 142.29\left(\mathrm{~s}, \mathrm{C}_{6} \mathrm{H}_{4}\right)$; IR $\left(\mathrm{ATR} \mathrm{cm}^{-1}\right)$ 3397, 2957, 2926, 1613, 1525, 1516 , $1466,1310,1228,1183,1107,816 . \mathrm{Mp} 125{ }^{\circ} \mathrm{C}$ to $127{ }^{\circ} \mathrm{C}$. Anal. Calcd for $\mathrm{C}_{26} \mathrm{H}_{32} \mathrm{~N}_{2}$ : C, 83.81; H, 8.67. Found: C, 83.55: H, 8.52.

(2) 1,4-bis( $N$-4'-iodophenyl,- $N$-4"'-n-butylphenylamino)benzene. A $250 \mathrm{~mL}$ Schlenk tube 
was charged with $3.72 \mathrm{~g}(0.010 \mathrm{~mol})$ of $\mathbf{1}, 33 \mathrm{~g}(0.10 \mathrm{~mol})$ of 1,4-diiodobenzene, $73 \mathrm{mg}(0.10$ $\mathrm{mmol})$ of $\mathrm{Pd}(\mathrm{DPPF}) \mathrm{Cl}_{2}, 1.92 \mathrm{~g}(0.02 \mathrm{~mol})$ of $\mathrm{Nat}-\mathrm{OBu}$, in a dry box. $50 \mathrm{~mL}$ of toluene was added via a cannula under nitrogen, and the reaction mixture was allowed to stir at $110{ }^{\circ} \mathrm{C}$ for 3 days. The reaction mixture became red after about $30 \mathrm{~min}$ of heating, followed by the formation of precipitate. The reaction was quenched by the addition of water $(150 \mathrm{~mL})$. The reaction mixture was extracted with chloroform $(3 \times 50 \mathrm{~mL})$. The organic solvent was removed by evaporation to leave a dark tar. The tar was dissolved in ethyl acetate and purified by column chromatography (silica gel in 10 to 1 mixture of hexanes and chloroform) to yield light yellow crystals of $2(1.53 \mathrm{~g}, 20 \%) .{ }^{1} \mathrm{H}$ NMR $\left(400 \mathrm{MHz}, \mathrm{CDCl}_{3}\right): \delta 0.87(\mathrm{t}, J=$ 7.32, 6H, $\mathrm{CH}_{3}$ ), 1.26 (mult, 4H, $\mathrm{CH}_{2}$ ), 1.48 (mult, 4H, $\mathrm{CH}_{2}$ ), 2.42 (t, J=7.72, 4H, $\mathrm{CH}_{2}$ ), 4.75(s, 2H, NH), 6.98 (mult, 20H, $\left.\mathrm{C}_{6} \mathrm{H}_{4}\right) ;{ }^{13} \mathrm{C}\left(100 \mathrm{MHz}, \mathrm{C}_{6} \mathrm{D}_{6}\right) \delta 13.67\left(\mathrm{~s}, \mathrm{CH}_{3}\right), 22.22\left(\mathrm{~s}, \mathrm{CH}_{2}\right), 33.59$ (s, $\left.\mathrm{CH}_{2}\right), 34.93\left(\mathrm{~s}, \mathrm{CH}_{2}\right), 84.12\left(\mathrm{~s}, \mathrm{C}_{6} \mathrm{H}_{4}\right), 124.53\left(\mathrm{~s}, \mathrm{C}_{6} \mathrm{H}_{4}\right), 124.85\left(\mathrm{~s}, \mathrm{C}_{6} \mathrm{H}_{4}\right), 125.17$ (s, $\left.\mathrm{C}_{6} \mathrm{H}_{4}\right)$, $129.38\left(\mathrm{~s}, \mathrm{C}_{6} \mathrm{H}_{4}\right), 138.01\left(\mathrm{~s}, \mathrm{C}_{6} \mathrm{H}_{4}\right), 138.12\left(\mathrm{~s}, \mathrm{C}_{6} \mathrm{H}_{4}\right), 142.67\left(\mathrm{~s}, \mathrm{C}_{6} \mathrm{H}_{4}\right), 144.89\left(\mathrm{~s}, \mathrm{C}_{6} \mathrm{H}_{4}\right), 147.79$ $\left(\mathrm{s}, \mathrm{C}_{6} \mathrm{H}_{4}\right)$; IR (ATR cm $\mathrm{cm}^{-1}$ ) 2958, 2927, 2858, 1579, 1502, 1482, 1311, 1276, 1181, 1115, 1063 , 999, 833, 815, 698. Mp $185{ }^{\circ} \mathrm{C}$ to $16.5{ }^{\circ} \mathrm{C}$. Anal. Calcd for $\mathrm{C}_{38} \mathrm{H}_{38} \mathrm{~N}_{2} \mathrm{I}_{2}: \mathrm{C}, 58.77 ; \mathrm{H}, 4.94$. Found: C, 58.82: H, 5.18.

(3) $N, N$-Bis-p-bromophenyl-p-anisidine. A $250 \mathrm{~mL} \mathrm{Schlenk} \mathrm{tube} \mathrm{was} \mathrm{charged} \mathrm{with} 6.16 \mathrm{~g}$ $(0.05 \mathrm{~mol})$ of anisidine, $35.4 \mathrm{~g}(0.15 \mathrm{~mol})$ of 1,4-dibromo-benzene, $0.73 \mathrm{~g}(1.0 \mathrm{mmol})$ of $\mathrm{Pd}(\mathrm{DPPF}) \mathrm{Cl}_{2}$ and $10.0 \mathrm{~g}(0.104 \mathrm{~mol})$ of $t$-BuONa in a dry box. $30 \mathrm{~mL}$ of toluene was added via a cannula under nitrogen and the reaction mixture was allowed to stir at $100{ }^{\circ} \mathrm{C}$ for 2 days. The reaction mixture became brick-red after 30 min of stirring, followed by the formation of precipitate. The reaction was quenched by the addition of water $(200 \mathrm{~mL})$. The reaction mixture was extracted with chloroform $(3 \times 50 \mathrm{~mL})$ and the organic solvent was removed by evaporation to leave a dark tar. The tar was dissolved in ethyl acetate and purified by column chromatography (silica gel in 9 to 1 mixture of hexanes and ethyl acetate) to yield white crystals of $\mathbf{3}(13 \mathrm{~g}, 61 \%) .{ }^{1} \mathrm{H}$ NMR $\left(400 \mathrm{MHz}, \mathrm{CDCl}_{3}\right): \delta 3.77\left(\mathrm{~s}, 3 \mathrm{H}, \mathrm{OCH}_{3}\right), 6.81$ (d, J=9.2, $\left.4 \mathrm{H} \mathrm{C}_{6} \mathrm{H}_{4}\right), 6.86\left(\mathrm{~d}, J=8.8,4 \mathrm{H} \mathrm{C}_{6} \mathrm{H}_{4}\right), 7.00\left(\mathrm{~d}, J=9.2,2 \mathrm{H}, \mathrm{C}_{6} \mathrm{H}_{4}\right), 7.27\left(\mathrm{~d}, J=8.8,2 \mathrm{H}, \mathrm{C}_{6} \mathrm{H}_{4}\right)$; ${ }^{13} \mathrm{C}\left(100 \mathrm{MHz}, \mathrm{CDCl}_{3}\right) \delta 55.56\left(\mathrm{~s}, \mathrm{OCH}_{3}\right), 114.61\left(\mathrm{~s}, \mathrm{C}_{6} \mathrm{H}_{4}\right), 115.10\left(\mathrm{~s}, \mathrm{C}_{6} \mathrm{H}_{4}\right), 124.33\left(\mathrm{~s}, \mathrm{C}_{6} \mathrm{H}_{4}\right)$, $127.48\left(\mathrm{~s}, \mathrm{C}_{6} \mathrm{H}_{4}\right), 132.23\left(\mathrm{~s}, \mathrm{C}_{6} \mathrm{H}_{4}\right), 139.78\left(\mathrm{~s}, \mathrm{C}_{6} \mathrm{H}_{4}\right), 146.88\left(\mathrm{~s}, \mathrm{C}_{6} \mathrm{H}_{4}\right), 156.79\left(\mathrm{~s}, \mathrm{C}_{6} \mathrm{H}_{4}\right) ; \mathrm{IR}$ 
(ATR cm-1) 2958, 2931, 2908, 2833, 2360, 2341, 1577, 1506, 1483, 1438, 1309, 1285, 1242, 1178, 1103, 1071, 1033, 1004, 822. Mp $70.5^{\circ} \mathrm{C}$ to $72^{\circ} \mathrm{C}$. Anal. Calcd for $\mathrm{C}_{19} \mathrm{H}_{15} \mathrm{Br}_{2} \mathrm{NO}$ : $\mathrm{C}$, 52.68; H, 3.50. Found: C, 53.12: H, 3.76.

(4) $N$-p-bromophenyl- $N$-p-iodophenyl-p-anisidine. A $250 \mathrm{~mL}$ Schlenk tube was charged with $16.25 \mathrm{~g} \quad(0.050 \mathrm{~mol})$ of $N$-p-iodophenyl aniline, $66 \mathrm{~g} \quad(0.20 \mathrm{~mol})$ of 1-bromo-4-iodo-benzene, $0.73 \mathrm{~g}(1.0 \mathrm{mmol})$ of $\mathrm{Pd}(\mathrm{DPPF}) \mathrm{Cl}_{2}$ and $10.0 \mathrm{~g}(0.104 \mathrm{~mol})$ of $t$-BuONa in a dry box. $30 \mathrm{~mL}$ of toluene was added via a cannula under nitrogen and the reaction mixture was allowed to stir at $100{ }^{\circ} \mathrm{C}$ for 5 days. The reaction mixture became brick red after a half an hour followed by the formation of precipitate. The reaction was quenched by the addition of water $(200 \mathrm{~mL})$ and extracted with chloroform $(3 \times 100 \mathrm{~mL})$. The organic solvent was removed by evaporation leaving a dark tar. The tar was dissolved in ethyl acetate and purified by column chromatography (silica gel in 2 to 1 mixture of hexanes and chloroform) to yield 4 as a low melting point solid (5.03 g, $21 \%) .{ }^{1} \mathrm{H}$ NMR (400 MHz, $\left.\mathrm{CDCl}_{3}\right)$ : $\delta 3.81\left(\mathrm{~s}, 3 \mathrm{H}, \mathrm{OCH}_{3}\right), 6.81\left(\mathrm{~d}, J=8.8,2 \mathrm{H} \mathrm{C}_{6} \mathrm{H}_{4}\right), 6.87\left(\mathrm{~d}, J=8.8,2 \mathrm{H}, \mathrm{C}_{6} \mathrm{H}_{4}\right), 6.93(\mathrm{~d}, J=8.8$, $\left.2 \mathrm{H}, \mathrm{C}_{6} \mathrm{H}_{4}\right), 7.06\left(\mathrm{~d}, J=8.8,2 \mathrm{H}, \mathrm{C}_{6} \mathrm{H}_{4}\right) 7.32\left(\mathrm{~d}, J=8.8,2 \mathrm{H}, \mathrm{C}_{6} \mathrm{H}_{4}\right), 7.49\left(\mathrm{~d}, J=8.8,2 \mathrm{H}, \mathrm{C}_{6} \mathrm{H}_{4}\right) ;$ ${ }^{13} \mathrm{C}\left(100 \mathrm{MHz}, \mathrm{CDCl}_{3}\right) \delta 55.49\left(\mathrm{~s}, \mathrm{OCH}_{3}\right), 77.30\left(\mathrm{~s}, \mathrm{C}_{6} \mathrm{H}_{4}\right), 84.68\left(\mathrm{~s}, \mathrm{C}_{6} \mathrm{H}_{4}\right), 114.82\left(\mathrm{~s}, \mathrm{C}_{6} \mathrm{H}_{4}\right)$, $115.17\left(\mathrm{~s}, \mathrm{C}_{6} \mathrm{H}_{4}\right), 124.47\left(\mathrm{~s}, \mathrm{C}_{6} \mathrm{H}_{4}\right), 127.45\left(\mathrm{~s}, \mathrm{C}_{6} \mathrm{H}_{4}\right), 132.21\left(\mathrm{~s}, \mathrm{C}_{6} \mathrm{H}_{4}\right), 138.10\left(\mathrm{~s}, \mathrm{C}_{6} \mathrm{H}_{4}\right)$, $139.59\left(\mathrm{~s}, \mathrm{C}_{6} \mathrm{H}_{4}\right), 146.66\left(\mathrm{~s}, \mathrm{C}_{6} \mathrm{H}_{4}\right), 147.51\left(\mathrm{~s}, \mathrm{C}_{6} \mathrm{H}_{4}\right), 156.89\left(\mathrm{~s}, \mathrm{C}_{6} \mathrm{H}_{4}\right)$; IR $\left(\right.$ ATR $\left.\mathrm{cm}^{-1}\right)$ 2964, 2927, 2835, 1576, 1506, 1483, 1313, 1284, 1242, 1178, 1104, 1072, 1035, 1004, 818, 756. Mp $74{ }^{\circ} \mathrm{C}$ to $76{ }^{\circ} \mathrm{C}$. Anal. Calcd for $\mathrm{C}_{19} \mathrm{H}_{15}$ BrINO: C, 47.52; H, 3.16. Found: C, 47.65: H, 3.38.

(5) 1,4-bis $\left(\boldsymbol{P}-\mathbf{2}^{\prime}\right.$, , $^{\prime}, 5$ '-trimethylpentylphosphino)benzene. A $100 \mathrm{~mL}$ Schlenk tube was charged with $45 \mathrm{mg}(0.20 \mathrm{mmol})$ of $\mathrm{Pd}(\mathrm{OAc})_{2}, 4.92 \mathrm{~g}(44 \mathrm{mmol})$ of DABCO, and $6.6 \mathrm{~g}(20$ mmol) of 1,4-diiodobenzene in a dry box, $40 \mathrm{~mL}$ of toluene was added via a cannula under nitrogen followed by stirring until the reaction mixture became homogeneous then $5.84 \mathrm{~g}(0.04$ mmol) 2,4,4-trimthylpentylphosphine was added via a syringe under nitrogen. The reaction mixture was allowed to stir at $60{ }^{\circ} \mathrm{C}$ for 24 hours. The reaction mixture became dark red in 5 min followed by the formation of precipitate. The reaction mixture was cooled to room temperature and the organic phase separated from the precipitate via filtration. The residual 
precipitate was washed with three portions of toluene $(20 \mathrm{~mL})$. Combined organic phases were concentrated yielding 5 as a colorless oil (3.5 g $48 \%$ yield). ${ }^{1} \mathrm{H}$ NMR (400 MHz, $\left.\mathrm{CDCl}_{3}\right): \delta$ $0.83\left(\mathrm{~s}, 18 \mathrm{H}, \mathrm{C}\left(\mathrm{CH}_{3}\right)_{3}\right), 0.99\left(\mathrm{~d}, J=6.6 \mathrm{~Hz}, 6 \mathrm{H}, \mathrm{CH}_{3}\right), 1.12\left(\mathrm{dd}, J=5.5,15.9 \mathrm{~Hz}, \quad 4 \mathrm{H}, \mathrm{CH}_{2}\right)$, 1.33(dd, $J=3.6,15.9 \mathrm{~Hz}, 2 \mathrm{H}, \mathrm{CH}_{2}$ ), 1.65 (mult, 2H, CH), 1.71 (mult, $2 \mathrm{H}, \mathrm{PCH}_{2}$ ), 1.82 (mult, 2H, $\mathrm{PCH}_{2}$ ), 4.09 (br, 2H, $\mathrm{PH}$ ), 7.39 (mult, 4H, $\left.\mathrm{C}_{6} \mathrm{H}_{4}\right) ;{ }^{13} \mathrm{C}\left(100 \mathrm{MHz}, \mathrm{CDCl}_{3}\right) \delta 23.68\left(\mathrm{~s}, \mathrm{CH}_{3}\right)$, $28.66\left(\mathrm{~s}, \mathrm{PCH}_{2}\right), 30.09$ (s, $\left.\mathrm{C}\left(\mathrm{CH}_{3}\right)_{3}\right), 31.22\left(\mathrm{~s}, \mathrm{C}\left(\mathrm{CH}_{3}\right)_{3}\right), 33.99\left(\mathrm{~s}, \mathrm{CH}_{2}\right), 52.32(\mathrm{~s}, \mathrm{CH}), 133.52$ (s, $\left.\mathrm{C}_{6} \mathrm{H}_{4}\right), 136.18\left(\mathrm{~s}, \mathrm{C}_{6} \mathrm{H}_{4}\right) ;{ }^{31} \mathrm{P}\left(162 \mathrm{MHz}, \mathrm{CDCl}_{3}\right) \delta-60.47$ (s, PH), IR $\left(\mathrm{ATR} \mathrm{cm}{ }^{-1}\right) 3054,2954$, 2901, 2867, 2285, 1475, 1414, 1377, 1364, 1248, 1199, 1117, 1052, 1017, 912, 804, 503. Anal. Calcd for $\mathrm{C}_{22} \mathrm{H}_{40} \mathrm{P}_{2}$ : C, 72.08; H, 11.02. Found: C, 71.20: H, 11.07.

(6a) A 3 dram vial was charged with $0.776 \mathrm{~g}(1.00 \mathrm{mmol})$ of $2,0.146 \mathrm{~g}(1.00 \mathrm{mmol})$ of 2,4,4-trimethylpentyl phosphine, $23 \mathrm{mg}(0.02 \mathrm{mmol})$ of $\mathrm{Pd}\left(\mathrm{PPh}_{3}\right)_{4}, 0.30 \mathrm{~g}(2.7 \mathrm{mmol})$ of DABCO and a stir flee. The vial was sealed with a Teflon cap and heated at $130{ }^{\circ} \mathrm{C}$ for 7 days. Upon heating, the reaction mixture became a dark viscous oil, followed by solidification after 24 hours. After the reaction was complete, $3.0 \mathrm{~mL}$ of THF was added to the reaction mixture which was sonicated for $6 \mathrm{~h}$. to suspend the product. The reaction mixture was then precipitated into rapidly stirring methanol to yield $0.96 \mathrm{~g}(1.5 \mathrm{mmol}, 75 \%)$ of $\mathbf{6 a}$ as light yellow powder. ${ }^{1} \mathrm{H}$ NMR (400 MHz, $\mathrm{CDCl}_{3}$ ): $\delta 0.79$ (mult, $12 \mathrm{H}, \mathrm{CH}_{3}$ ), 1.05 (mult, br, $3 \mathrm{H}$, $\mathrm{CH}_{3}$ ), 1.33 (br, 6H, $\mathrm{CH}_{2}$ ), 1.56 (br, 5H, $\mathrm{CH}$ and $\mathrm{CH}_{2}$ ), 1.83 (br, $1 \mathrm{H}, \mathrm{PCH}_{2}$ ), 1.98 (br, $1 \mathrm{H}$, $\left.\mathrm{PCH}_{2}\right), 2.54$ (br, 4H, $\left.\mathrm{CH}_{2}\right), 6.95\left(\mathrm{br}, 12 \mathrm{H}, \mathrm{C}_{6} \mathrm{H}_{4}\right) ;{ }^{13} \mathrm{C}\left(100 \mathrm{MHz}, \mathrm{CDCl}_{3}\right): \delta 14.15\left(\mathrm{br}, \mathrm{CH}_{3}\right)$,

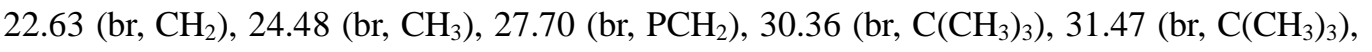
33.81 (br, $\mathrm{CH}_{2}$ ), 35.17 (br, $\mathrm{CH}_{2}$ ), 40.53 (br, $\mathrm{CH}_{2}$ ), 53.27 (br, $\mathrm{CH}$ ), 122.21 (br, $\mathrm{C}_{6} \mathrm{H}_{4}$ ), 125.22 (br, $\mathrm{C}_{6} \mathrm{H}_{4}$ ), 125.78 (br, $\mathrm{C}_{6} \mathrm{H}_{4}$ ), 129.49 (br, $\mathrm{C}_{6} \mathrm{H}_{4}$ ), 133.64 (br, $\mathrm{C}_{6} \mathrm{H}_{4}$ ), 134.20 (br, $\mathrm{C}_{6} \mathrm{H}_{4}$ ), 138.37 (br, $\left.\mathrm{C}_{6} \mathrm{H}_{4}\right), 143.05\left(\mathrm{br}, \mathrm{C}_{6} \mathrm{H}_{4}\right), 145.29\left(\mathrm{br}, \mathrm{C}_{6} \mathrm{H}_{4}\right), 148.52\left(\mathrm{br}, \mathrm{C}_{6} \mathrm{H}_{4}\right) ;{ }^{31} \mathrm{P}\left(162 \mathrm{MHz}, \mathrm{CDCl}_{3}\right) \delta 22.37$ (s, $\mathrm{PR}_{3}$ ), -61.95 (s, PH) , -62.53 (s, PH), for the soluble fraction IR (ATR $\mathrm{cm}^{-1}$ ) 2955, 2931, $2872,1734,1589,1501,1315,1266,1111,1072,1018,827,723$. For the insoluble fraction: 2954, 2931, 2870, 1735, 1589, 1501, 1314, 1267, 1109, 1018, 825, 733; SEC (THF vs polystyrene standards) $\mathrm{M}_{\mathrm{n}}=5000, \mathrm{PDI}=1.6$.

(6b) A suspension of $100 \mathrm{mg}(0.140 \mathrm{mmol})$ of $6 \mathbf{a}$ and $5 \mathrm{~mL}$ of ethanol was stirred at room 
temperature. $110 \mathrm{mg}(1 \mathrm{mmol})$ of a $30 \% \mathrm{H}_{2} \mathrm{O}_{2}$ solution was added slowly, to the suspension. The addition of $\mathrm{H}_{2} \mathrm{O}_{2}$ did not result in significant visual changes to the suspension. The suspension was stirred overnight, followed by evaporation of all of the solvent. The residue was extracted with $2 \mathrm{~mL} \mathrm{CHCl}_{3}$, then precipitated in hexane to yield $88 \mathrm{mg}$ of $\mathbf{6 b}$ as a brown powder (0.120mmol 86\% yield). ${ }^{1} \mathrm{H} \mathrm{NMR}\left(400 \mathrm{MHz}, \mathrm{CDCl}_{3}\right): \delta 0.82\left(\mathrm{~s}, 9 \mathrm{H} \mathrm{C}\left(\mathrm{CH}_{3}\right)_{3}\right), 0.94$ (br, 6H CH 3 ), 1.12 (br, 3H CH 3 ), 1.41 (br, 4H CH ), 1.70 (br, 4H CH (mult, $1 \mathrm{H} \mathrm{PCH}_{2}$ ), 2.25 (mult, 1H PCH ), 2.59 (br, 4 $\mathrm{H} \mathrm{CH}_{2}$ ), 7.06 (br, $16 \mathrm{H} \mathrm{C}_{6} \mathrm{H}_{4}$ ); 7.55 (br, 4H $\left.\mathrm{C}_{6} \mathrm{H}_{4}\right) ; \delta^{13} \mathrm{C}\left(100 \mathrm{MHz}, \mathrm{CDCl}_{3}\right) 14.15\left(\mathrm{br}, \mathrm{CH}_{3}\right), 22.61$ (br, $\left.\mathrm{CH}_{2}\right), 24.77$ (br, $\left.\mathrm{CH}_{3}\right), 30.31$ (br, $\left.\mathrm{C}\left(\mathrm{CH}_{3}\right)_{3}\right), 31.46\left(\mathrm{br}, \mathrm{C}\left(\mathrm{CH}_{3}\right)_{3}\right), 33.74\left(\mathrm{br}, \mathrm{CH}_{2}\right), 35.33$ (br, $\left.\mathrm{CH}_{2}\right), 39.95\left(\mathrm{~d} J=104 \mathrm{~Hz}, \mathrm{PCH}_{2}\right)$, 53.55 (br, CH), 120.13 (br, $\mathrm{C}_{6} \mathrm{H}_{4}$ ), 124.51 (br, $\mathrm{C}_{6} \mathrm{H}_{4}$ ), 126.07 (br, $\mathrm{C}_{6} \mathrm{H}_{4}$ ), 129.74 (br, $\mathrm{C}_{6} \mathrm{H}_{4}$ ), 132.27 (br, $\mathrm{C}_{6} \mathrm{H}_{4}$ ), 139.44 (br, $\mathrm{C}_{6} \mathrm{H}_{4}$ ), 142.66 (br, $\mathrm{C}_{6} \mathrm{H}_{4}$ ), 144.29 (br, $\mathrm{C}_{6} \mathrm{H}_{4}$ ), 147.77 (br, $\mathrm{C}_{6} \mathrm{H}_{4}$ ), $151.08\left(\mathrm{br}, \mathrm{C}_{6} \mathrm{H}_{4}\right) ; \delta^{31} \mathrm{P}\left(162 \mathrm{MHz}, \mathrm{CDCl}_{3}\right) 31.84\left(\mathrm{~s}, \mathrm{PR}_{3}\right)$. SEC (THF vs polystyrene standards) $\mathrm{M}_{\mathrm{n}}=2200, \mathrm{PDI}=1.6$.

(8a) A 3 dram vial was charged with $0.479 \mathrm{~g}(1.00 \mathrm{mmol})$ of $4,0.146 \mathrm{~g}(1.00 \mathrm{mmol})$ of 2,4,4-trimethylpentyl phosphine, $23 \mathrm{mg}(0.02 \mathrm{mmol})$ of $\mathrm{Pd}\left(\mathrm{PPh}_{3}\right)_{4}, 0.30 \mathrm{~g}(2.7 \mathrm{mmol})$ of $\mathrm{DABCO}$ and a stir flee. The vial was sealed with a Teflon cap and heated at $60{ }^{\circ} \mathrm{C}$ for 4 hours, followed by increasing the temperature to $130{ }^{\circ} \mathrm{C}$ and stirring for another 7 days. Upon heating, the reaction mixture became a dark viscous oil followed by solidification after 24 hours. After the reaction was complete, $3.0 \mathrm{~mL}$ of THF was added to the reaction mixture, which was sonicated for $6 \mathrm{~h}$. to suspend the product. The reaction mixture was then precipitated into rapidly stirring methanol to yield $0.38 \mathrm{~g}(0.91 \mathrm{mmol}, 91 \%)$ of $\mathbf{8 a}$ as gray powder. ${ }^{1} \mathrm{H}$ NMR (400 MHz, $\mathrm{CDCl}_{3}$ ): $\delta 0.79$ (mult, 9H, $\left.\mathrm{C}\left(\mathrm{CH}_{3}\right)_{3}\right), 1.06$ (br, 3H, $\mathrm{CH}_{3}$ ), 1.14(mult, 1H, $\mathrm{CH}_{2}$ ), 1.41 (mult, 1H, $\mathrm{CH}_{2}$ ), 1.56 (br, 1H, $\mathrm{CH}$ ), 1.88 (mult, 1H, $\mathrm{PCH}_{2}$ ), 2.03 (mult,1H, $\mathrm{PCH}_{2}$ ), $3.78\left(\mathrm{~s}, 3 \mathrm{H}, \mathrm{OCH}_{3}\right), 6.95\left(\right.$ mult, $\left.12 \mathrm{H}, \mathrm{C}_{6} \mathrm{H}_{4}\right) ;{ }^{13} \mathrm{C}\left(100 \mathrm{MHz}, \mathrm{CDCl}_{3}\right) \delta$ 24.48 (br, $\mathrm{CH}_{3}$ ), 27.45 (br, $\mathrm{PCH}_{2}$ ), 30.27 (br, $\left.\mathrm{C}\left(\mathrm{CH}_{3}\right)_{3}\right), 31.46$ (br, $\left.\mathrm{C}\left(\mathrm{CH}_{3}\right)_{3}\right), 40.30$ (br, $\mathrm{CH}_{2}$ ), 53.32 (br, CH), 55.68 (br, OCH), 115.51 (br, $\mathrm{C}_{6} \mathrm{H}_{4}$ ), 122.55 (br, $\mathrm{C}_{6} \mathrm{H}_{4}$ ), 128.23 (br, $\mathrm{C}_{6} \mathrm{H}_{4}$ ), 131.50 (br, $\left.\mathrm{C}_{6} \mathrm{H}_{4}\right), 140.18$ (br, $\left.\mathrm{C}_{6} \mathrm{H}_{4}\right), 148.27$ (br, $\left.\mathrm{C}_{6} \mathrm{H}_{4}\right), 156.86$ (br, $\left.\mathrm{C}_{6} \mathrm{H}_{4}\right) ;{ }^{31} \mathrm{P}(162 \mathrm{MHz}$, $\left.\mathrm{CDCl}_{3}\right) \delta$-21.73 (s, $\mathrm{PR}_{3}$ ), -62.04 (s, PH); IR (ATR cm ${ }^{-1}$ ) (soluble fraction) 2954, 2904, 2870, $1582,1507,1494,1318,1288,1243,1182,1103,1069,1038,912,826$, (insoluble fraction) 
2952, 2902, 2868, 1582, 1507, 1494, 1364, 1317, 1287, 1242, 1182, 1105, 1037, 824, 731; SEC (THF vs polystyrene standards) $M_{n}=3000, P D I=1.5$.

(8b) A suspension of $100 \mathrm{mg}(0.214 \mathrm{mmol})$ of $\mathbf{8 a}$ and $5 \mathrm{~mL}$ of ethanol was stirred at room temperature. $110 \mathrm{mg}(1 \mathrm{mmol})$ of a $30 \% \mathrm{H}_{2} \mathrm{O}_{2}$ solution was added slowly. The suspension became clear upon the addition of the $\mathrm{H}_{2} \mathrm{O}_{2}$. The mixture was stirred overnight, followed by evaporation of all of the solvent. The residue was extracted with $2 \mathrm{mLCHCl}_{3}$ then precipitated in hexane to yield $83 \mathrm{mg}$ of $\mathbf{8 b}$ as a brown powder ( $0.172 \mathrm{mmol} 80 \%$ yield). ${ }^{1} \mathrm{H}$ NMR (400 $\mathrm{MHz}, \mathrm{CDCl}_{3}$ ): $\delta 0.82$ (br, 9H C( $\left.\left(\mathrm{CH}_{3}\right)_{3}\right), 1.10$ (br, $3 \mathrm{H} \mathrm{CH}_{3}$ ), 1.18 (mult, $1 \mathrm{H} \mathrm{CH}_{2}$ ), 1.40 (mult, $1 \mathrm{H}$ $\mathrm{CH}_{2}$ ), 1.92 (br, $1 \mathrm{H} \mathrm{CH}$ ), 2.10 (mult, $1 \mathrm{H} \mathrm{PCH}_{2}$ ), 2.21 (mult, $1 \mathrm{H} \mathrm{PCH}$ ), 3.82 (s, $3 \mathrm{H} \mathrm{OCH}_{3}$ ), 6.88 (br, 2 $\left.\mathrm{H} \mathrm{C}_{6} \mathrm{H}_{4}\right), 7.07$ (br, $\left.6 \mathrm{H} \mathrm{C}_{6} \mathrm{H}_{4}\right) 7.58\left(\mathrm{br}, 4 \mathrm{H} \mathrm{C}_{6} \mathrm{H}_{4}\right) ; \delta^{13} \mathrm{C}\left(100 \mathrm{MHz}, \mathrm{CDCl}_{3}\right) 24.77\left(\mathrm{~s}, \mathrm{CH}_{2}\right)$, 25.19 (br, $\left.\mathrm{CH}_{3}\right), 30.26\left(\mathrm{~s}, \mathrm{C}\left(\mathrm{CH}_{3}\right)_{3}\right), 31.49\left(\mathrm{~s}, \mathrm{C}\left(\mathrm{CH}_{3}\right)_{3}\right), 39.90\left(\mathrm{~d} J=70 \mathrm{~Hz} \mathrm{PCH}_{2}\right), 53.48$ (br, $\mathrm{CH}), 55.73\left(\mathrm{~s}, \mathrm{OCH}_{3}\right), 115.67\left(\mathrm{br}, \mathrm{C}_{6} \mathrm{H}_{4}\right), 122.21\left(\mathrm{br}, \mathrm{C}_{6} \mathrm{H}_{4}\right), 126.82\left(\mathrm{~d} J=118 \mathrm{~Hz} \mathrm{C}_{6} \mathrm{H}_{4}\right), 128.77$ (br, $\mathrm{C}_{6} \mathrm{H}_{4}$ ), 132.59 (br, $\mathrm{C}_{6} \mathrm{H}_{4}$ ), 139.08 (br, $\mathrm{C}_{6} \mathrm{H}_{4}$ ), 150.30 (br, $\mathrm{C}_{6} \mathrm{H}_{4}$ ), 157.77 (br, $\mathrm{C}_{6} \mathrm{H}_{4}$ ); $\delta^{31} \mathrm{P}$ $\left(162 \mathrm{MHz}, \mathrm{CDCl}_{3}\right) 32.18\left(\mathrm{~s}, \mathrm{PR}_{3}\right) . \quad \mathrm{SEC}\left(\mathrm{THF}\right.$ vs polystyrene standards) $\mathrm{M}_{\mathrm{n}}=1500, \mathrm{PDI}=$ 1.5 .

(9a) A 3 dram vial was charged with $0.732 \mathrm{~g}(2.00 \mathrm{mmol})$ of $\mathbf{5}, 0.866 \mathrm{~g}(2.00 \mathrm{mmol})$ of $\mathbf{3}, 46 \mathrm{mg}$ $(0.04 \mathrm{mmol})$ of $\mathrm{Pd}\left(\mathrm{PPh}_{3}\right)_{4}, 0.50 \mathrm{~g}(4.5 \mathrm{mmol})$ of $\mathrm{DABCO}$ and a stir flee. The vial was sealed with a Teflon cap and heated at $130{ }^{\circ} \mathrm{C}$ for 7 days. Upon heating, the reaction mixture became a dark viscous oil followed by solidification after 24 hours. After the reaction was complete, $2.0 \mathrm{~mL}$ of THF was added to the reaction mixture which was sonicated for $6 \mathrm{~h}$. to suspend the product. The reaction mixture was then precipitated into rapidly stirring methanol to yield $1.03 \mathrm{~g}(1.61 \mathrm{mmol}, 80.6 \%)$ of $9 \mathrm{a}$ as brown powder. ${ }^{1} \mathrm{H} \mathrm{NMR}\left(400 \mathrm{MHz}, \mathrm{CDCl}_{3}\right): \delta 0.77(\mathrm{~s}$, $\left.18 \mathrm{H}, \mathrm{C}\left(\mathrm{CH}_{3}\right)_{3}\right), 1.05$ (br, 6H, $\mathrm{CH}_{3}$ ), 1.15 (mult, 2H, $\mathrm{CH}_{2}$ ), 1.425 (mult, 2H, $\mathrm{CH}_{2}$ ), 1.52 (br, $2 \mathrm{H}$, $\mathrm{CH}$ ), 1.86 (mult, 2H, $\mathrm{PCH}_{2}$ ), 2.00 (mult, $2 \mathrm{H}, \mathrm{PCH}_{2}$ ), 3.77 (s, 3H, $\mathrm{OCH}_{3}$ ), 7.15 (mult, $16 \mathrm{H}$, $\left.\mathrm{C}_{6} \mathrm{H}_{4}\right) ;{ }^{13} \mathrm{C}\left(100 \mathrm{MHz}, \mathrm{CDCl}_{3}\right) \delta 24.37\left(\mathrm{br}, \mathrm{CH}_{3}\right), 27.23\left(\mathrm{br}, \mathrm{PCH}_{2}\right), 30.12\left(\mathrm{~s}, \mathrm{C}\left(\mathrm{CH}_{3}\right)_{3}\right), 31.33$ (s, $\left.\mathrm{C}\left(\mathrm{CH}_{3}\right)_{3}\right), 39.79$ (br, $\left.\mathrm{CH}_{2}\right), 33.59$ (s, $\left.\mathrm{CH}_{2}\right), 52.74$ (br, $\left.\mathrm{CH}\right), 55.55$ (s, $\left.\mathrm{OCH}_{3}\right), 115.08$ (br, $\mathrm{C}_{6} \mathrm{H}_{4}$ ), 122.57 (br, $\mathrm{C}_{6} \mathrm{H}_{4}$ ), 128.24 (mult, $\mathrm{C}_{6} \mathrm{H}_{4}$ ), 130.06 (br, $\mathrm{C}_{6} \mathrm{H}_{4}$ ), 132.38 (mult, $\mathrm{C}_{6} \mathrm{H}_{4}$ ), 134.32 (mult, $\left.\mathrm{C}_{6} \mathrm{H}_{4}\right), 139.84\left(\mathrm{br}, \mathrm{C}_{6} \mathrm{H}_{4}\right), 148.26\left(\mathrm{br}, \mathrm{C}_{6} \mathrm{H}_{4}\right), 156.86\left(\mathrm{br}, \mathrm{C}_{6} \mathrm{H}_{4}\right),{ }^{31} \mathrm{P}\left(162 \mathrm{MHz}, \mathrm{CDCl}_{3}\right) \delta$ 
-21.93 (s, $\mathrm{PR}_{3}$ ), -20.91(s, $\mathrm{PR}_{3}$ ), IR (ATR cm ${ }^{-1}$ ) 2954, 2908, 2873, 1586, 1500, 1321, 1294, 1244 , $1186,1120,1038,914,828,804,737$; SEC (THF vs polystyrene standards) $M_{n}=10,400$, PDI $=2.0$.

(9b) A suspension of $100 \mathrm{mg}(0.136 \mathrm{mmol})$ of $9 \mathbf{a}$ and $5 \mathrm{~mL}$ of ethanol was stirred at room temperature. $110 \mathrm{mg}(1 \mathrm{mmol})$ of a $30 \% \mathrm{H}_{2} \mathrm{O}_{2}$ solution was added slowly. The suspension became clear upon the addition of the $\mathrm{H}_{2} \mathrm{O}_{2}$. The mixture was stirred overnight, followed by evaporation of all of the solvent. The residue was extracted with $2 \mathrm{~mL} \mathrm{CHCl}_{3}$ then precipitated in hexane to yield $85 \mathrm{mg}$ of $\mathbf{9 b}$ as a brown powder $\left(0.11 \mathrm{mmol} 82 \%\right.$ yield). ${ }^{1} \mathrm{H}$ NMR (400 $\left.\mathrm{MHz}, \mathrm{CDCl}_{3}\right): \delta 0.81\left(\mathrm{~s}, 18 \mathrm{H} \mathrm{C}\left(\mathrm{CH}_{3}\right)_{3}\right), 1.07\left(\mathrm{br}, 6 \mathrm{H} \mathrm{CH}_{3}\right), 1.17\left(\mathrm{br}, 2 \mathrm{H} \mathrm{CH}_{2}\right), 1.38(\mathrm{br}, 2 \mathrm{H}$ $\mathrm{CH}_{2}$ ), 2.10 (br, $2 \mathrm{H} \mathrm{CH}$ ), 2.15 (mult, $2 \mathrm{H} \mathrm{PCH}_{2}$ ), 2.26 (mult, $2 \mathrm{H} \mathrm{PCH}$ ), 3.80 (s, 3H OCH$)_{3}$ ), 6.88 (br, 2H C $\left.\mathrm{C}_{6} \mathrm{H}_{4}\right), 7.07\left(\mathrm{br}, 6 \mathrm{H} \mathrm{C}_{6} \mathrm{H}_{4}\right) 7.55\left(\mathrm{br}, 4 \mathrm{H} \mathrm{C}_{6} \mathrm{H}_{4}\right) 7.85\left(\mathrm{br}, 4 \mathrm{H} \mathrm{C}_{6} \mathrm{H}_{4}\right) ; \delta^{13} \mathrm{C}(100 \mathrm{MHz}$, $\left.\mathrm{CDCl}_{3}\right) 24.37\left(\mathrm{~d} J=12 \mathrm{~Hz}, \mathrm{CH}_{2}\right), 25.14\left(\mathrm{br}, \mathrm{CH}_{3}\right), 30.20\left(\mathrm{~s}, \mathrm{C}\left(\mathrm{CH}_{3}\right)_{3}\right), 31.33\left(\mathrm{~s}, \mathrm{C}\left(\mathrm{CH}_{3}\right)_{3}\right)$, $39.49\left(\mathrm{~d} J=70 \mathrm{~Hz} \mathrm{PCH}_{2}\right), 53.30(\mathrm{br}, \mathrm{CH}), 55.71\left(\mathrm{~s}, \mathrm{OCH}_{3}\right), 115.51\left(\mathrm{br}, \mathrm{C}_{6} \mathrm{H}_{4}\right), 122.22(\mathrm{br}$, $\left.\mathrm{C}_{6} \mathrm{H}_{4}\right), 125.80\left(\mathrm{~d} J=82 \mathrm{~Hz} \mathrm{C} \mathrm{C}_{6} \mathrm{H}_{4}\right), 128.78\left(\mathrm{br}, \mathrm{C}_{6} \mathrm{H}_{4}\right), 130.90\left(\mathrm{br}, \mathrm{C}_{6} \mathrm{H}_{4}\right), 132.12$ (br, $\left.\mathrm{C}_{6} \mathrm{H}_{4}\right)$ 137.74((d $\left.J=45 \mathrm{~Hz} \mathrm{C} \mathrm{C}_{6} \mathrm{H}_{4}\right), 138.66\left(\mathrm{br}, \mathrm{C}_{6} \mathrm{H}_{4}\right), 150.30$ (br, $\left.\mathrm{C}_{6} \mathrm{H}_{4}\right), 157.91$ (br, $\left.\mathrm{C}_{6} \mathrm{H}_{4}\right), \delta^{31} \mathrm{P}$ $\left(162 \mathrm{MHz}, \mathrm{CDCl}_{3}\right) 31.01\left(\mathrm{~s}, \mathrm{PR}_{3}\right) . \quad$ SEC (THF vs polystyrene standards) $\mathrm{M}_{\mathrm{n}}=5000, \mathrm{PDI}=$ 1.8 .

(10a) $N, N$-Bis- $p$-(diphenylphosphino)phenyl-p-anisidine. A 3 dram vial was charged with $0.433 \mathrm{~g}(1.00 \mathrm{mmol})$ of $3,0.372 \mathrm{~g}(2.00 \mathrm{mmol})$ of diphenylphosphine, $23 \mathrm{mg}(0.02 \mathrm{mmol})$ of $\mathrm{Pd}\left(\mathrm{PPh}_{3}\right)_{4}, \quad 0.30 \mathrm{~g}(2.7 \mathrm{mmol})$ of DABCO and a stir flee. The vial was sealed with a Teflon cap and heated at $130{ }^{\circ} \mathrm{C}$ for 14 days. Upon heating, the reaction mixture became a yellow viscous oil. After the reaction was complete, the reaction mixture was extracted with warm hexanes $(3 \times 10 \mathrm{~mL})$ and recrystallized from hexane in a freezer $\left(-30{ }^{\circ} \mathrm{C}\right)$ to yield $0.29 \mathrm{~g}(0.45$ mmol $45 \%$ yield $)$ of $\mathbf{1 0 a}$ as light yellow powder. ${ }^{1} \mathrm{H} \mathrm{NMR}\left(400 \mathrm{MHz}, \mathrm{CDCl}_{3}\right): \delta 3.74(\mathrm{~s}, 3 \mathrm{H}$, $\left.\mathrm{OCH}_{3}\right), 7.03$ (mult, $\left.32 \mathrm{H}, \mathrm{C}_{6} \mathrm{H}_{4}\right) ;{ }^{13} \mathrm{C}$ NMR $\left(100 \mathrm{MHz}, \mathrm{CDCl}_{3}\right): \delta 55.68\left(\mathrm{~s}, \mathrm{OCH}_{3}\right), 115.20(\mathrm{~s}$, $\left.\mathrm{C}_{6} \mathrm{H}_{4}\right), 122.45\left(\mathrm{~d}, J=7, \mathrm{C}_{6} \mathrm{H}_{4}\right), 128.50\left(\mathrm{~s}, \mathrm{C}_{6} \mathrm{H}_{4}\right), 128.60\left(\mathrm{~s}, \mathrm{C}_{6} \mathrm{H}_{5}\right), 128.72\left(\mathrm{~d}, J=9, \mathrm{C}_{6} \mathrm{H}_{5}\right)$, $129.15\left(\mathrm{~d}, J=7, \mathrm{C}_{6} \mathrm{H}_{5}\right), 133.77\left(\mathrm{~d}, J=19, \mathrm{C}_{6} \mathrm{H}_{5}\right), 135.07\left(\mathrm{~d}, J=19, \mathrm{C}_{6} \mathrm{H}_{4}\right), 137.94(\mathrm{~d}, J=10$, $\left.\mathrm{C}_{6} \mathrm{H}_{4}\right), 139.78\left(\mathrm{~s}, \mathrm{C}_{6} \mathrm{H}_{4}\right), 148.38\left(\mathrm{~s}, \mathrm{C}_{6} \mathrm{H}_{4}\right), 157.17\left(\mathrm{~s}, \mathrm{C}_{6} \mathrm{H}_{4}\right) ;{ }^{31} \mathrm{P}$ NMR $\left(162 \mathrm{MHz}, \mathrm{CDCl}_{3}\right) \delta$ 
$-3.26\left(\mathrm{~s}, \mathrm{PR}_{3}\right) ; \quad$ IR $\left(\mathrm{ATR} \mathrm{cm}^{-1}\right): 3052,1581,1506,1493,1434,1317,1289,1242,1182,1094$, 1031, 826, 743. Anal. Calcd for $\mathrm{C}_{43} \mathrm{H}_{35} \mathrm{NOP}_{2}: \mathrm{C}, 80.22: \mathrm{H}, 5.49 ; \mathrm{N}, 2.18$. Found: C, 79.91: H, $5.47 ; \mathrm{N}, 1.98$.

(10b) A suspension of $100 \mathrm{mg}(0.135 \mathrm{mmol})$ of $\mathbf{1 0 a}$ and $5 \mathrm{~mL}$ of ethanol was stirred at room temperature. $110 \mathrm{mg}(1 \mathrm{mmol})$ of a $30 \% \mathrm{H}_{2} \mathrm{O}_{2}$ solution was added slowly. The suspension became clear upon the addition of the $\mathrm{H}_{2} \mathrm{O}_{2}$. The mixture was stirred overnight, followed by evaporation of all of the solvent. The residue was dissolved in $1 \mathrm{ml}$ methanol and purified by column chromatography (silica gel in 1 to 1 mixture of hexanes and methanol) to yield $53 \mathrm{mg}$ of $\mathbf{1 0 b}$ as a brown powder (0.055 mmol, $\left.41 \%) .{ }^{1} \mathrm{H} \mathrm{NMR} \mathrm{(400} \mathrm{MHz}, \mathrm{CDCl}_{3}\right): \delta 3.63(\mathrm{~s}, 3 \mathrm{H}$ $\left.\mathrm{OCH}_{3}\right), 7.18$ (mult, $\left.32 \mathrm{H} \mathrm{C}_{6} \mathrm{H}_{4}\right) ; \delta^{13} \mathrm{C}\left(100 \mathrm{MHz}, \mathrm{CDCl}_{3}\right)$ 55.31(s, $\left.\mathrm{OCH}_{3}\right), 115.19\left(\mathrm{~s}, \mathrm{C}_{6} \mathrm{H}_{4}\right)$, $121.50\left(\mathrm{~d}, J=12, \mathrm{C}_{6} \mathrm{H}_{4}\right), 124.91\left(\mathrm{~d}, J=109, \mathrm{C}_{6} \mathrm{H}_{5}\right), 128.31\left(\mathrm{~d}, J=12, \mathrm{C}_{6} \mathrm{H}_{5}\right), 128.63\left(\mathrm{~s}, \mathrm{C}_{6} \mathrm{H}_{4}\right)$, $131.71\left(\mathrm{~s}, \mathrm{C}_{6} \mathrm{H}_{5}\right), 131.81$ (d, $\left.J=9, \mathrm{C}_{6} \mathrm{H}_{5}\right), 132.55$ (d, J=109, $\left.\mathrm{C}_{6} \mathrm{H}_{4}\right), 133.15$ (d, J=10, $\mathrm{C}_{6} \mathrm{H}_{4}$ ), $138.15\left(\mathrm{~s}, \mathrm{C}_{6} \mathrm{H}_{4}\right), 149.97\left(\mathrm{~s}, \mathrm{C}_{6} \mathrm{H}_{4}\right), 157.59\left(\mathrm{~s}, \mathrm{C}_{6} \mathrm{H}_{4}\right) ; \delta^{31} \mathrm{P}\left(162 \mathrm{MHz}, \mathrm{CDCl}_{3}\right) 29.10\left(\mathrm{~s}, \mathrm{PR}_{3}\right)$. Anal. Calcd for $\mathrm{C}_{43} \mathrm{H}_{35} \mathrm{NO}_{3} \mathrm{P}_{2}: \mathrm{C}, 76.43: \mathrm{H}, 5.23 ; \mathrm{N}, 2.07$. Found: C, 76.08: H, 5.23; N, 1.83.

\section{(11a)1,4-bis( $N$-4'-(diphenylphosphino)phenyl,-N-4"'-n-butylphenylamino)benzene.}

A 3 dram vial was charged with $0.777 \mathrm{~g}(1.00 \mathrm{mmol})$ of $2,0.372 \mathrm{~g}(2.00 \mathrm{mmol})$ of diphenylphosphine, $26 \mathrm{mg}(0.02 \mathrm{mmol})$ of $\mathrm{Pd}\left(\mathrm{PPh}_{3}\right)_{4} \quad 0.3 \mathrm{~g}(2.7 \mathrm{mmol})$ of DABCO and a stir flee. The vial was sealed with a Teflon cap and heated at $130{ }^{\circ} \mathrm{C}$ for 2 days. Upon heating, the reaction mixture became a viscous yellow oil. After the reaction was complete, the reaction mixture was extracted with warm hexane $(3 \times 10 \mathrm{~mL})$ and recrystallized from hexane in a freezer $\left(-30{ }^{\circ} \mathrm{C}\right)$ to yield $0.58 \mathrm{~g}(0.65 \mathrm{mmol} 65 \%$ yield $)$ of $11 \mathrm{a}$ as a light yellow powder. ${ }^{1} \mathrm{H}$ NMR (400 MHz, $\mathrm{CDCl}_{3}$ ): $\delta 0.83$ (t, $J=7.32,6 \mathrm{H}, \mathrm{CH}_{3}$ ), 1.26 (mult, 4H, $\mathrm{CH}_{2}$ ), 1.48 (pent, $J=$ 7.72, 4H, $\mathrm{CH}_{2}$ ), 2.46(t, $\left.J=7.32,4 \mathrm{H}, \mathrm{CH}_{2}\right), 7.04$ (mult, $\left.40 \mathrm{H}, \mathrm{C}_{6} \mathrm{H}_{4}, \mathrm{C}_{6} \mathrm{H}_{5}\right) ;{ }^{13} \mathrm{C}(100 \mathrm{MHz}$, $\left.\mathrm{CDCl}_{3}\right): \delta 14.17\left(\mathrm{~s}, \mathrm{CH}_{3}\right), 22.59\left(\mathrm{~s}, \mathrm{CH}_{2}\right), 33.80\left(\mathrm{~s}, \mathrm{CH}_{2}\right), 35.26\left(\mathrm{~s}, \mathrm{CH}_{2}\right), 121.38$ (d, J =7.7 $\left.\mathrm{C}_{6} \mathrm{H}_{4}\right), 125.57\left(\mathrm{~s}, \mathrm{C}_{6} \mathrm{H}_{4}\right), 126.00\left(\mathrm{~s}, \mathrm{C}_{6} \mathrm{H}_{4}\right), 128.58\left(\mathrm{~d}, J=7.7, \mathrm{C}_{6} \mathrm{H}_{5}\right), 128.63\left(\mathrm{~s}, \mathrm{C}_{6} \mathrm{H}_{5}\right), 129.10$ (d, $\left.J=12.24, \mathrm{C}_{6} \mathrm{H}_{4}\right), 129.53\left(\mathrm{~s}, \mathrm{C}_{6} \mathrm{H}_{4}\right), 133.71\left(\mathrm{~d}, J=19.1, \mathrm{C}_{6} \mathrm{H}_{5}\right), 135.04\left(\mathrm{~d}, J=21.4, \mathrm{C}_{6} \mathrm{H}_{4}\right)$, $138.15\left(\mathrm{~d}, J=10.7, \mathrm{C}_{6} \mathrm{H}_{5}\right), 138.71\left(\mathrm{~s}, \mathrm{C}_{6} \mathrm{H}_{4}\right), 142.86\left(\mathrm{~s}, \mathrm{C}_{6} \mathrm{H}_{4}\right), 144.80\left(\mathrm{~s}, \mathrm{C}_{6} \mathrm{H}_{4}\right) ; 148.96(\mathrm{~s}$, 
$\left.\mathrm{C}_{6} \mathrm{H}_{4}\right) ;{ }^{31} \mathrm{P}\left(162 \mathrm{MHz}, \mathrm{CDCl}_{3}\right): \delta-2.70\left(\mathrm{~s}, \mathrm{PR}_{3}\right) . \mathrm{IR}\left(\mathrm{ATR} \mathrm{cm}{ }^{-1}\right): 3051,2955,2927,2856,1588$, $1558,1541,1495,1434,1314,1268,1182,1094,824,742$. Anal. Calcd for $\mathrm{C}_{62} \mathrm{H}_{58} \mathrm{~N}_{2} \mathrm{P}_{2}: \mathrm{C}$, 83.37; H, 6.56; N, 3.14. Found: C, 82.98; H, 6.56; N, 2.94 .

(11b) A suspension of $100 \mathrm{mg}(0.101 \mathrm{mmol})$ of $11 \mathrm{a}$ and $5 \mathrm{~mL}$ of ethanol was stirred at room temperature. $110 \mathrm{mg}(1 \mathrm{mmol})$ of a $30 \% \mathrm{H}_{2} \mathrm{O}_{2}$ solution was added slowly. The suspension became clear upon the addition of the $\mathrm{H}_{2} \mathrm{O}_{2}$. The mixture was stirred overnight, followed by evaporationof all of the solvent. The residue was dissolved in $1 \mathrm{~mL}$ methanol and purified by column chromatography (silica gel in 1 to 1 mixture of hexanes and methanol) to yield $53 \mathrm{mg}$ of $11 \mathrm{~b}$ as a brown powder $(0.052 \mathrm{mmol}, 52 \%) .{ }^{1} \mathrm{H} \mathrm{NMR}\left(400 \mathrm{MHz}, \mathrm{CDCl}_{3}\right): \delta 0.86(\mathrm{t}, J=7.32$ $6 \mathrm{H} \mathrm{CH}_{3}$ ), 1.29(mult, $J=7.324 \mathrm{H} \mathrm{CH}_{2}$ ), 1.51 (mult, $J=7.724 \mathrm{H} \mathrm{CH}_{2}$ ), $2.52\left(\mathrm{t}, J=7.324 \mathrm{H} \mathrm{CH}_{2}\right.$ ), 7.29(mult, $\left.40 \mathrm{H} \mathrm{C}_{6} \mathrm{H}_{4}, \mathrm{C}_{6} \mathrm{H}_{5}\right) ; \delta^{13} \mathrm{C}\left(100 \mathrm{MHz}, \mathrm{CDCl}_{3}\right) 14.74\left(\mathrm{~s}, \mathrm{CH}_{3}\right), 23.54\left(\mathrm{~s}, \mathrm{CH}_{2}\right), 34.88$ (s, $\left.\mathrm{CH}_{2}\right), 36.13\left(\mathrm{~s}, \mathrm{CH}_{2}\right), 120.53\left(\mathrm{~d}, J=10.7 \mathrm{C}_{6} \mathrm{H}_{4}\right), 124.23\left(\mathrm{~d}, J=109, \mathrm{C}_{6} \mathrm{H}_{4}\right), 127.81\left(\mathrm{~s}, \mathrm{C}_{6} \mathrm{H}_{4}\right)$, $128.37\left(\mathrm{~s}, \mathrm{C}_{6} \mathrm{H}_{4}\right), 130.10\left(\mathrm{~d}, J=9.2, \mathrm{C}_{6} \mathrm{H}_{5}\right), 131.28\left(\mathrm{~s}, \mathrm{C}_{6} \mathrm{H}_{5}\right), 133.17\left(\mathrm{~s}, \mathrm{C}_{6} \mathrm{H}_{4}\right) 133.25(\mathrm{~d}, J=$ $\left.12.2, \mathrm{C}_{6} \mathrm{H}_{5}\right), 134.45\left(\mathrm{~d}, J=9.2, \mathrm{C}_{6} \mathrm{H}_{4}\right), 135.02\left(\mathrm{~d}, J=104, \mathrm{C}_{6} \mathrm{H}_{5}\right), 141.62\left(\mathrm{~s}, \mathrm{C}_{6} \mathrm{H}_{4}\right), 144.40(\mathrm{~s}$ $\left.\mathrm{C}_{6} \mathrm{H}_{4}\right), 145.30\left(\mathrm{~s}, \mathrm{C}_{6} \mathrm{H}_{4}\right) ; 152.82\left(\mathrm{~s}, \mathrm{C}_{6} \mathrm{H}_{4}\right) ; \delta^{31} \mathrm{P}\left(162 \mathrm{MHz}, \mathrm{CDCl}_{3}\right) 29.66\left(\mathrm{~s}, \mathrm{PR}_{3}\right)$. Anal. Calcd for $\mathrm{C}_{62} \mathrm{H}_{58} \mathrm{~N}_{2} \mathrm{O}_{2} \mathrm{P}_{2}$ : C, 80.49; H, 6.33; N, 3.03. Found: C, 80.14; H, 6.37; N, 2.72 .

(12a) A 3 dram vial was charged with $0.503 \mathrm{~g}(1.05 \mathrm{mmol})$ of $2,0.73 \mathrm{~g}(0.50 \mathrm{mmol})$ of 2,4,4-trimthylpentylphosphine, $26 \mathrm{mg}(0.02 \mathrm{mmol})$ of $\mathrm{Pd}\left(\mathrm{PPh}_{3}\right)_{4} \quad 0.134 \mathrm{~g}(1.20 \mathrm{mmol})$ ofDABCO and a stir flee. The vial was sealed with a Teflon cap and heated at $80{ }^{\circ} \mathrm{C}$ for 2 days. Upon heating, the reaction mixture became a viscous orange oil. After the reaction was complete, the reaction mixture was extracted with chloroform, then purified by column chromatography (silica gel in pure chloroform) in a drybox to yield 12a as a low melting point

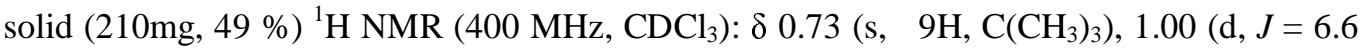
$\left.\mathrm{Hz}, 3 \mathrm{H}, \mathrm{CH}_{3}\right), 1.09\left(\mathrm{dd}, J=5.7,14 \mathrm{~Hz}, 1 \mathrm{H}, \mathrm{CH}_{2}\right), 1.33\left(\mathrm{dd}, J=3.3,14 \mathrm{~Hz}, 1 \mathrm{H}, \mathrm{CH}_{2}\right), 1.48$ (mult, $\mathrm{H}, \mathrm{CH}$ ) , 1.77 (mult, 1H, $\mathrm{PCH}_{2}$ ), 1.94 (mult, 1H, $\mathrm{PCH}_{2}$ ), 3.71 (s, 3H, $\mathrm{OCH}_{3}$ ), 6.95 (mult, $\left.24 \mathrm{H}, \mathrm{C}_{6} \mathrm{H}_{4}\right) ;{ }^{13} \mathrm{C}\left(100 \mathrm{MHz}, \mathrm{CDCl}_{3}\right): \delta 24.41\left(\mathrm{~d}, J=10 \mathrm{~Hz}, \mathrm{CH}_{3}\right), 27.36(\mathrm{~d}, J=12 \mathrm{~Hz}$, $\left.\mathrm{PCH}_{2}\right), 30.27$ (s, $\left.\mathrm{C}\left(\mathrm{CH}_{3}\right)_{3}\right), 31.45\left(\mathrm{~s}, \mathrm{C}\left(\mathrm{CH}_{3}\right)_{3}\right), 40.04\left(\mathrm{~s}, \mathrm{CH}_{2}\right), 52.99$ (d, J = 8.4 Hz, CH), $55.69\left(\mathrm{~s}, \mathrm{OCH}_{3}\right), 114.74\left(\mathrm{~d}, J=6.6 \mathrm{~Hz}, \mathrm{C}_{6} \mathrm{H}_{4}\right), 115.18\left(\mathrm{~s}, \mathrm{C}_{6} \mathrm{H}_{4}\right), 123.12(\mathrm{~d}, J=23 \mathrm{~Hz}$ 
$\left.\mathrm{C}_{6} \mathrm{H}_{4}\right), 124.83\left(\mathrm{~d}, J=10.8 \mathrm{~Hz}, \mathrm{C}_{6} \mathrm{H}_{4}\right), 127.87\left(\mathrm{~d}, J=9.9 \mathrm{~Hz}, \mathrm{C}_{6} \mathrm{H}_{4}\right), 132.29\left(\mathrm{~s}, \mathrm{C}_{6} \mathrm{H}_{4}\right), 133.76$ $\left(\mathrm{d}, J=19 \mathrm{~Hz}, \mathrm{C}_{6} \mathrm{H}_{4}\right), 134.18\left(\mathrm{~d}, J=20 \mathrm{~Hz}, \mathrm{C}_{6} \mathrm{H}_{4}\right), 140.00\left(\mathrm{~s}, \mathrm{C}_{6} \mathrm{H}_{4}\right), 147.06\left(\mathrm{~s}, \mathrm{C}_{6} \mathrm{H}_{4}\right)$, $148.19\left(\mathrm{~d}, J=23 \mathrm{~Hz}, \mathrm{C}_{6} \mathrm{H}_{4}\right), 156.93\left(\mathrm{~s}, \mathrm{C}_{6} \mathrm{H}_{4}\right),{ }^{31} \mathrm{P}\left(162 \mathrm{MHz}, \mathrm{CDCl}_{3}\right): \delta \quad-22.41\left(\mathrm{~s}, \mathrm{PR}_{3}\right)$. Anal. Calcd for $\mathrm{C}_{46} \mathrm{H}_{47} \mathrm{~N}_{2} \mathrm{O}_{2} \mathrm{PBr}_{2}$ : C, 64.94: H, 5.58: N, 3.29. Found: C, 64.54, H, 5.87: N, 3.14 .

(12b) A suspension of $85 \mathrm{mg}(0.10 \mathrm{mmol})$ of 12a and $5 \mathrm{~mL}$ of ethanol was stirred at room temperature. $55 \mathrm{mg}(0.5 \mathrm{mmol})$ of a $30 \% \mathrm{H}_{2} \mathrm{O}_{2}$ solution was added slowly, and the suspension became clear. The mixture was stirred overnight, followed by evaporation of all of the solvent. The residue was extracted with $2 \mathrm{~mL} \mathrm{CHCl}_{3}$, then recrystallized in acetonitrile to yield $55 \mathrm{mg}$ of $\mathbf{1 2 b}$ as a brown powder $\left(0.063 \mathrm{mmol} 63 \%\right.$ yield). ${ }^{1} \mathrm{H} \mathrm{NMR}\left(400 \mathrm{MHz}, \mathrm{CDCl}_{3}\right): \delta 0.82(\mathrm{~s}$, 9H, $\left.\mathrm{C}\left(\mathrm{CH}_{3}\right)_{3}\right), 1.09\left(\mathrm{~d}, J=6.4 \mathrm{~Hz}, 3 \mathrm{H}, \mathrm{CH}_{3}\right), 1.18\left(\mathrm{dd}, J=6.4,14 \mathrm{~Hz}, \quad 1 \mathrm{H}, \mathrm{CH}_{2}\right), 1.38(\mathrm{dd}, J=$ 3.2, $14 \mathrm{~Hz}, 1 \mathrm{H}, \mathrm{CH}_{2}$ ), 2.03 (mult, $\mathrm{H}, \mathrm{CH}$ ) , 2.04 (mult, 1H, $\mathrm{PCH}_{2}$ ), 2.17 (mult, 1H, $\mathrm{PCH}_{2}$ ), 3.71 (s, 3H, $\left.\mathrm{OCH}_{3}\right), 7.09$ (mult, 24H, $\left.\mathrm{C}_{6} \mathrm{H}_{4}\right) ;{ }^{13} \mathrm{C}\left(100 \mathrm{MHz}, \mathrm{CDCl}_{3}\right): \delta 24.74(\mathrm{~d}, J=4.5 \mathrm{~Hz}$, $\left.\mathrm{CH}_{3}\right), 25.18\left(\mathrm{~s}, \mathrm{CH}_{2}\right), 30.24\left(\mathrm{~s}, \mathrm{C}\left(\mathrm{CH}_{3}\right)_{3}\right), 31.45\left(\mathrm{~s}, \mathrm{C}\left(\mathrm{CH}_{3}\right)_{3}\right), 39.98(\mathrm{~d}, J=71 \mathrm{~Hz} \mathrm{CH}), 53.50$ $\left(\mathrm{d}, J=11 \mathrm{~Hz}, \mathrm{PCH}_{2}\right), 55.70\left(\mathrm{~s}, \mathrm{OCH}_{3}\right), 115.32\left(\mathrm{~s}, \mathrm{C}_{6} \mathrm{H}_{4}\right), 116.18\left(\mathrm{~d}, J=12 \mathrm{~Hz}, \mathrm{C}_{6} \mathrm{H}_{4}\right)$, $120.44\left(\mathrm{dd}, J=12,22 \mathrm{~Hz}, \mathrm{C}_{6} \mathrm{H}_{4}\right), 125.12\left(\mathrm{~d}, J=43 \mathrm{~Hz}, \mathrm{C}_{6} \mathrm{H}_{4}\right), 125.95\left(\mathrm{~d}, J=14 \mathrm{~Hz} \mathrm{C}_{6} \mathrm{H}_{4}\right)$, $128.22\left(\mathrm{~d}, J=10 \mathrm{~Hz} \mathrm{C} \mathrm{H}_{4}\right), 131.11$ (dd, $\left.J=10,18 \mathrm{~Hz}, \mathrm{C}_{6} \mathrm{H}_{4}\right), 132.55\left(\mathrm{~s}, \mathrm{C}_{6} \mathrm{H}_{4}\right), 139.32$ (s, $\left.\mathrm{C}_{6} \mathrm{H}_{4}\right), 146.30\left(\mathrm{~s}, \mathrm{C}_{6} \mathrm{H}_{4}\right), 150.61\left(\mathrm{~s}, \mathrm{C}_{6} \mathrm{H}_{4}\right), 157.35\left(\mathrm{~s}, \mathrm{C}_{6} \mathrm{H}_{4}\right) ;{ }^{31} \mathrm{P}\left(162 \mathrm{MHz}, \mathrm{CDCl}_{3}\right): \delta$ 33.06(s, $\mathrm{PR}_{3}$ ). Anal. Calcd for $\mathrm{C}_{46} \mathrm{H}_{47} \mathrm{~N}_{2} \mathrm{O}_{3} \mathrm{PBr}_{2}: \mathrm{C}, 63.74: \mathrm{H}$, 5.48: N, 3.23. Found: $\mathrm{C}, 63.56: \mathrm{H}$, 5.77: N, 3.14 .

(13b) A suspension of $110 \mathrm{mg}(0.50 \mathrm{mmol})$ of $\mathbf{1 3 a}$ and $5 \mathrm{~mL}$ of ethanol was stirred at room temperature. $110 \mathrm{mg}(1 \mathrm{mmol})$ of a $30 \% \mathrm{H}_{2} \mathrm{O}_{2}$ solution was added slowly, and the suspension became clear. The mixture was stirred overnight, followed by evacuation of all of the solvent. The residue was extracted with $2 \mathrm{~mL} \mathrm{CHCl}_{3}$, then precipitated in hexane to yield $75 \mathrm{mg}$ of $\mathbf{1 3 b}$ as a brown powder $(0.318 \mathrm{mmol} 64 \%$ yield $) .{ }^{1} \mathrm{H} \mathrm{NMR}\left(400 \mathrm{MHz}, \mathrm{CDCl}_{3}\right): \delta 0.76(\mathrm{br}, 9 \mathrm{H}$ 
$\left.\mathrm{C}\left(\mathrm{CH}_{3}\right)_{3}\right), 1.02\left(\mathrm{br}, 3 \mathrm{H} \mathrm{CH}_{3}\right), 1.19$ (br, $\left.1 \mathrm{H} \mathrm{CH}_{2}\right), 1.36$ (br, $\left.1 \mathrm{H} \mathrm{CH}_{2}\right), 2.03$ (br, 1H CH), 2.17 (br, $1 \mathrm{H} \mathrm{PCH}$ ), $2.30\left(\mathrm{br}, 1 \mathrm{H} \mathrm{PCH}\right.$ ), $7.84\left(\mathrm{br}, 4 \mathrm{H} \mathrm{C}_{6} \mathrm{H}_{4}\right) ; \delta^{13} \mathrm{C}\left(100 \mathrm{MHz}, \mathrm{CDCl}_{3}\right) 24.47\left(\mathrm{~s}, \mathrm{CH}_{3}\right)$, 25.03 (br, $\mathrm{CH}_{2}$ ), $29.98\left(\mathrm{~s}, \mathrm{C}\left(\mathrm{CH}_{3}\right)_{3}\right), 31.23$ (s, $\left.\mathrm{CH}\right), 38.88$ (d $\left.J=70 \mathrm{~Hz} \mathrm{PCH}_{2}\right), 53.03$ (br, $\left.\mathrm{C}\left(\mathrm{CH}_{3}\right)_{3}\right), 130.87\left(\mathrm{br}, \mathrm{C}_{6} \mathrm{H}_{4}\right), 137.69\left(\left(\mathrm{~d} J=93 \mathrm{~Hz} \mathrm{C}_{6} \mathrm{H}_{4}\right), \delta^{31} \mathrm{P}\left(162 \mathrm{MHz}, \mathrm{CDCl}_{3}\right) 30.23(\mathrm{~s}\right.$, $\left.\mathrm{PR}_{3}\right)$. 

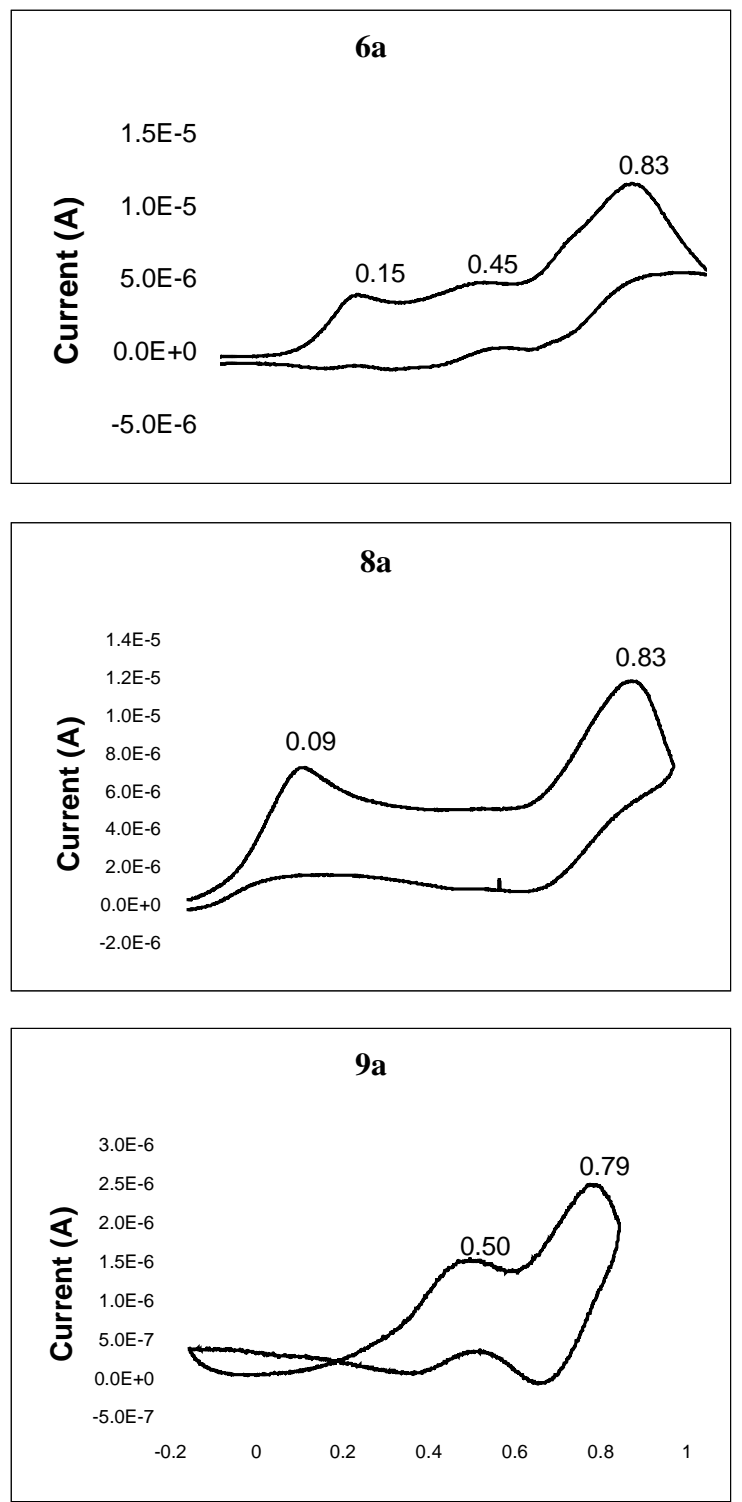

Electric Potential (V vs Fc/Fc $\left.{ }^{+}\right)$

Figure S1. (Top) Cyclic Voltammograph for polymer 6a. (Middle) Cyclic Voltammograph for polymer 8a (Bottom) Cyclic Voltammograph for polymer 9a. 

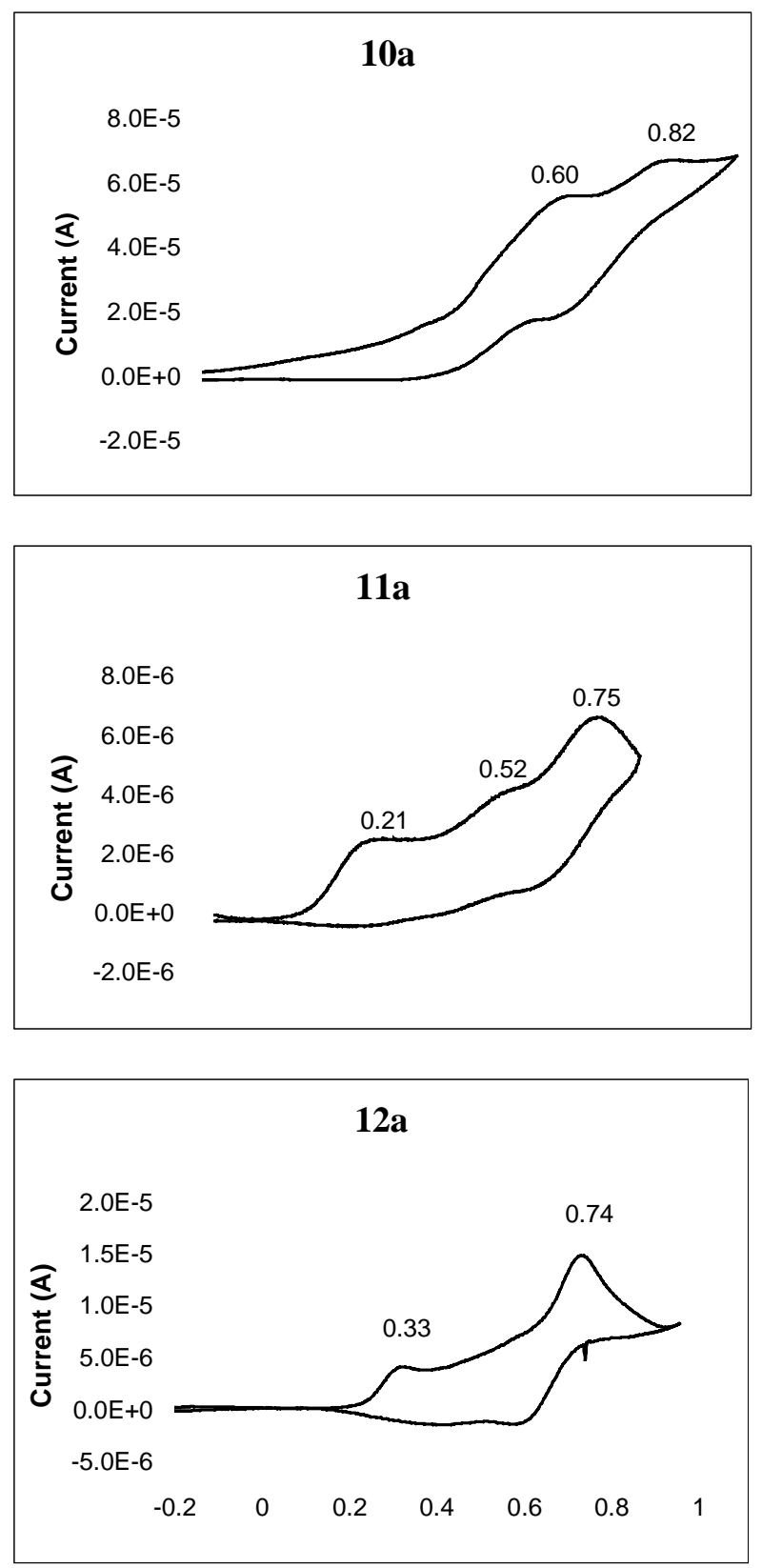

Electric Potential (V vs Fc/Fc ${ }^{+}$)

Figure S2. (Top) Cyclic Voltammograph for polymer model compounds 10a (Top), 11a (middle), and 12a (Bottom). 

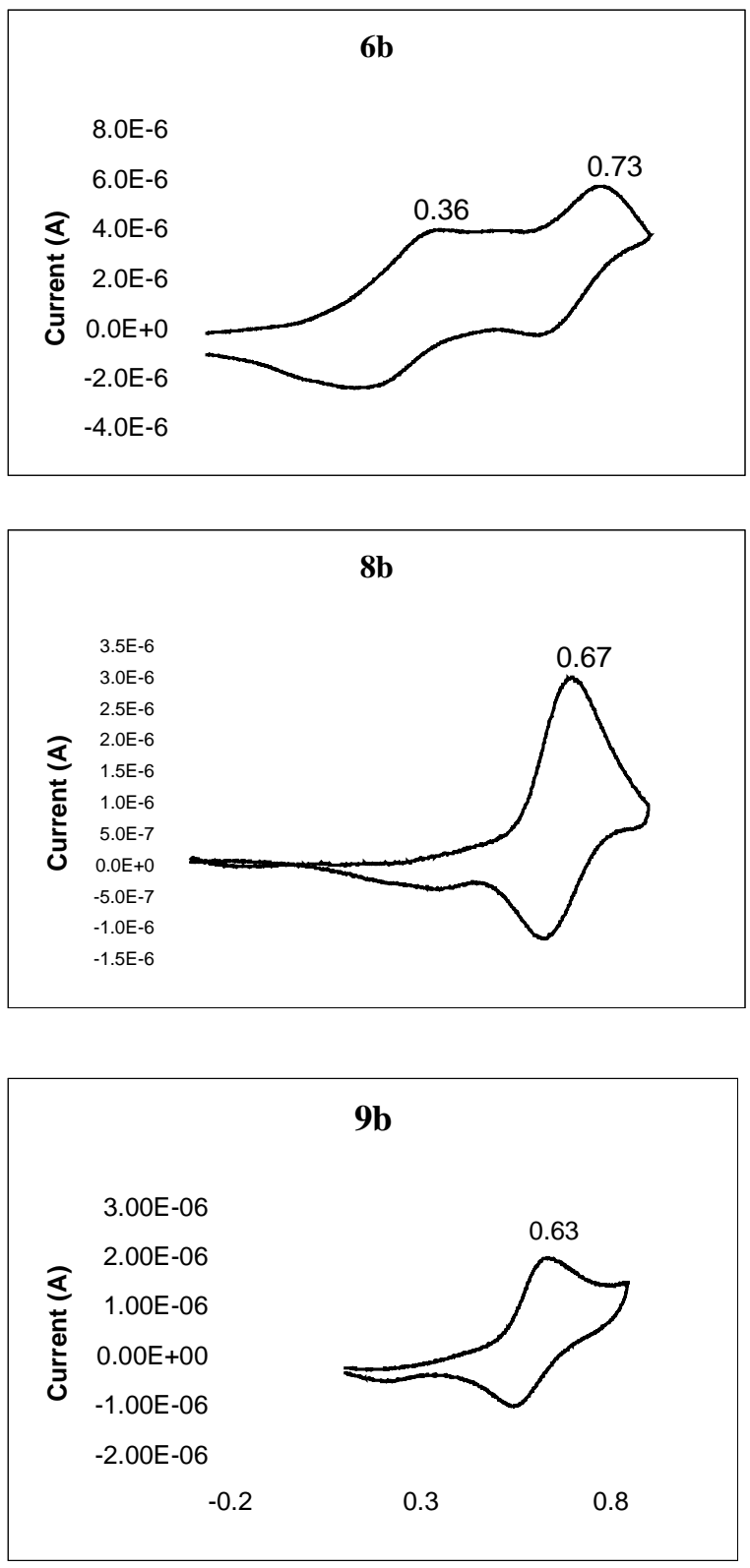

Electric Potential (V vs Fc/ $\left.\mathrm{Fc}^{+}\right)$

Figure S3. Cyclic Voltammographs for PPPP Oxide-PANI copolymers $\mathbf{6 b}$ (Top), $\mathbf{8 b}$ (Middle), and $\mathbf{9 b}$ (Bottom). 

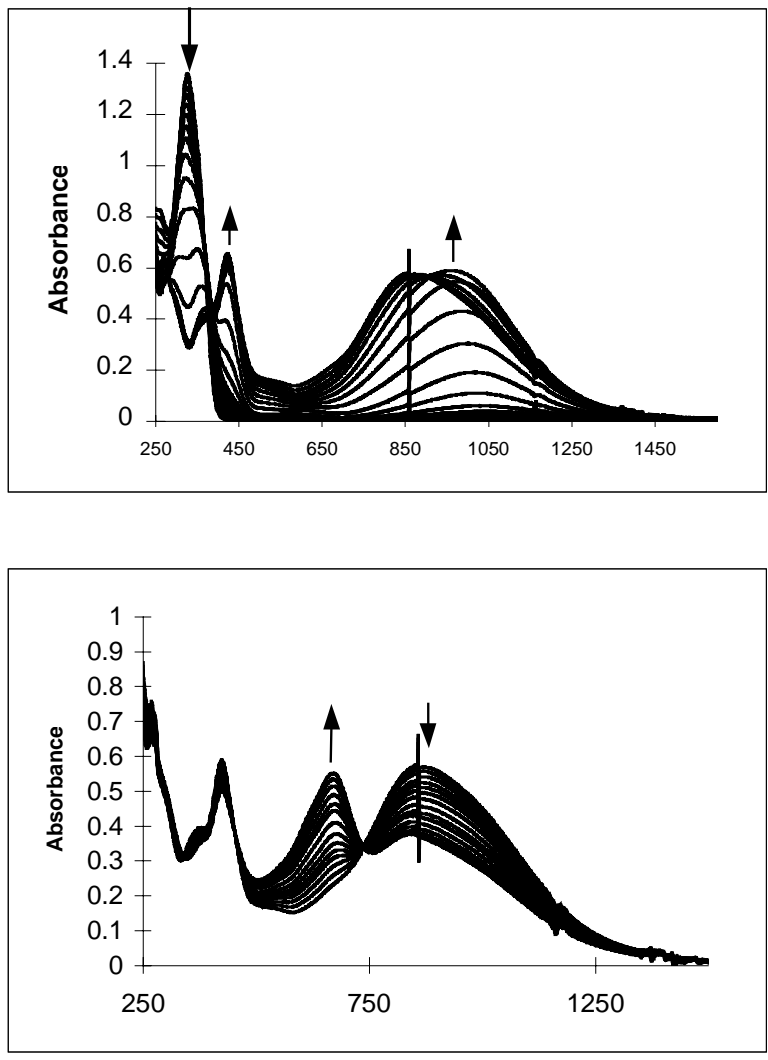

Wavelength (nm)

Figure S4. UV-visible spectra of the chemical oxidation of model compound 11a. $\left(2.05 \times 10^{-5} \mathrm{M}\right)$ with $\mathrm{NOBF}_{4}$ (0.75 equiv per aliquot). (Top) addition of 0.0 to 15 equiv. of $\mathrm{NOBF}_{4}$. (Bottom) addition of 15 to 25.5 equiv. of $\mathrm{NOBF}_{4}$. 

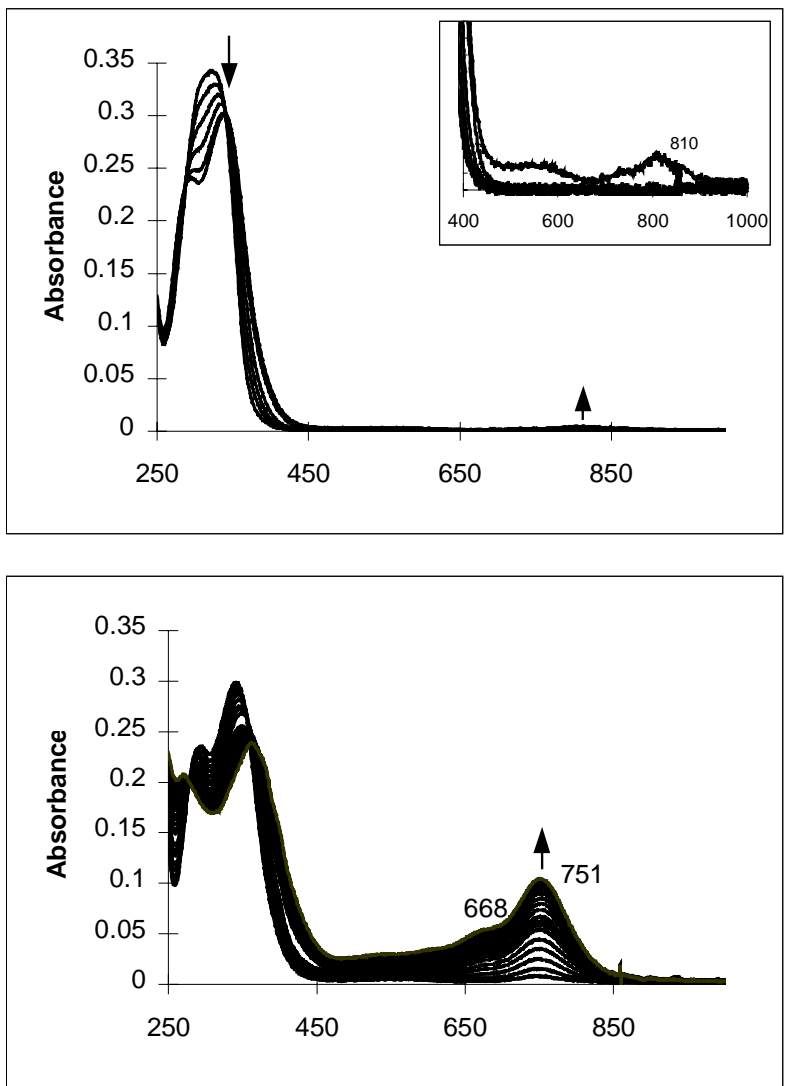

Wavelength (nm)

Figure S5. Chemical oxidation of 12a $\left(1.0 \times 10^{-5} \mathrm{M}\right)$ with $\mathrm{NOBF}_{4}$ (0.33 equiv per aliquot). (Top) Addition of 0.0 to 1.66 equiv. of $\mathrm{NOBF}_{4}$. (Bottom) Addition of 1.66 to 9.00 equiv. of $\mathrm{NOBF}_{4}$. 


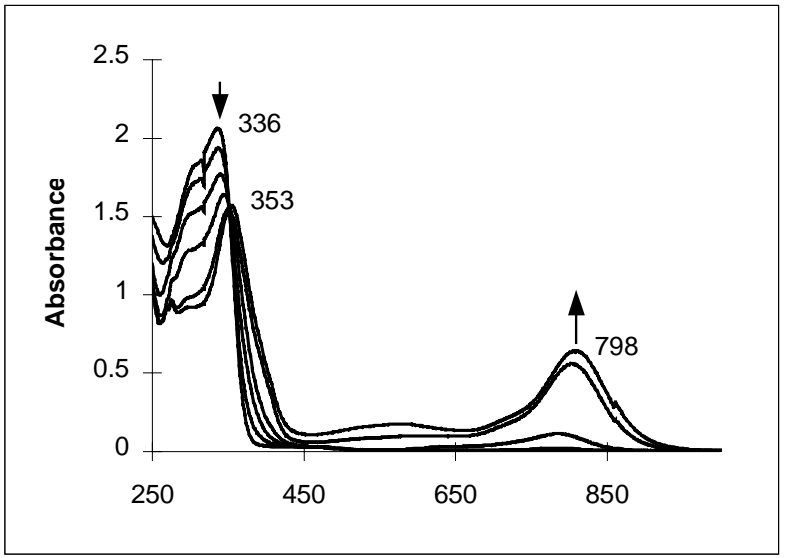

Wavelength (nm)

Figure S6. Chemical oxidation of model compound 10a. $\left(4.8 \times 10^{-5} \mathrm{M}\right)$ with $\mathrm{NOBF}_{4}(0.60$ equiv per aliquot $)$. Addition of 0.0 to 3 equiv. of $\mathrm{NOBF}_{4}$. 

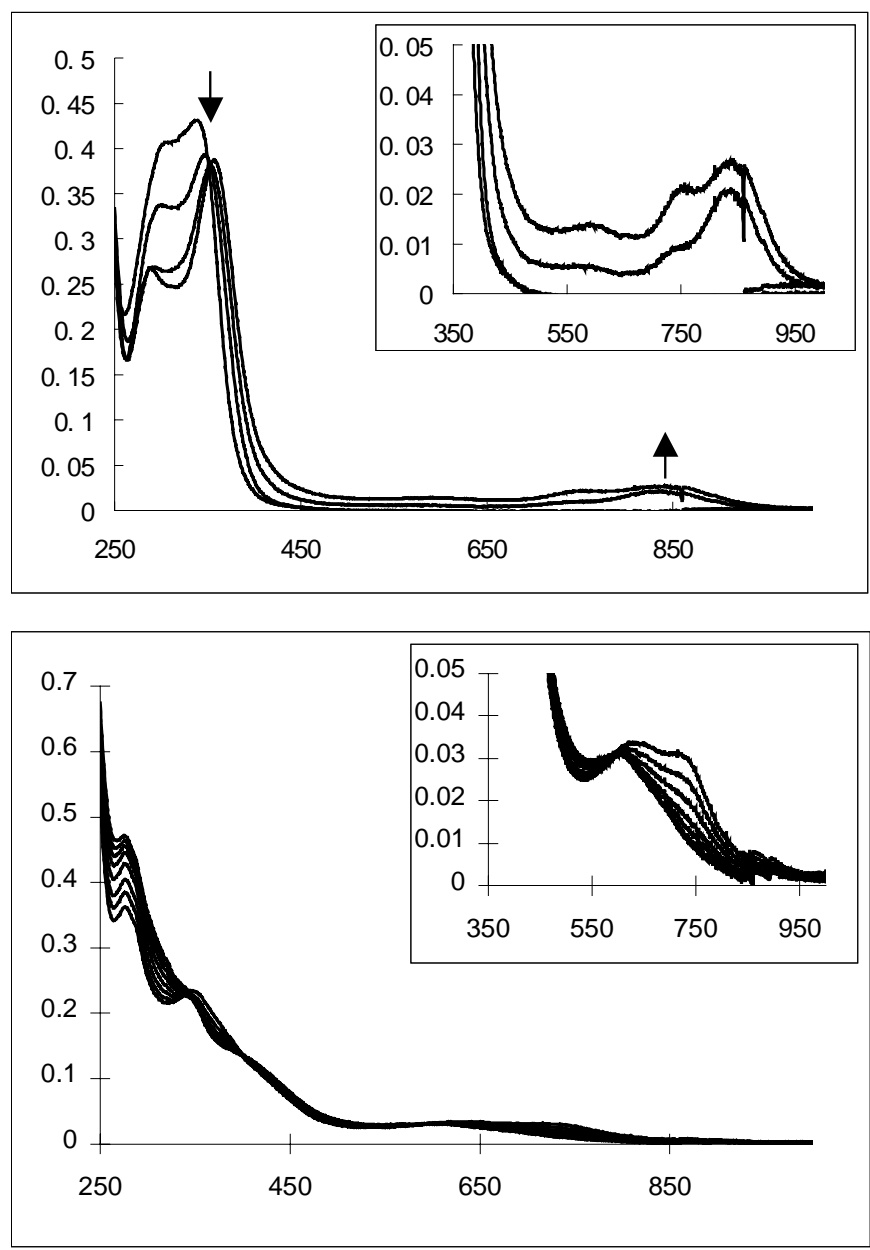

Figure S7. UV-Visible-NIR spectra of polymer 9a. $\quad\left(1.7 \times 10^{-5} \mathrm{M}\right)$ with $\mathrm{NOBF}_{4}$ (1.0 equiv per aliquot). (Top) Addition of 0.0 to 3.0 equiv. of $\mathrm{NOBF}_{4}$. (Bottom) Addition of 3.0 to 10 equiv. of $\mathrm{NOBF}_{4}$. 


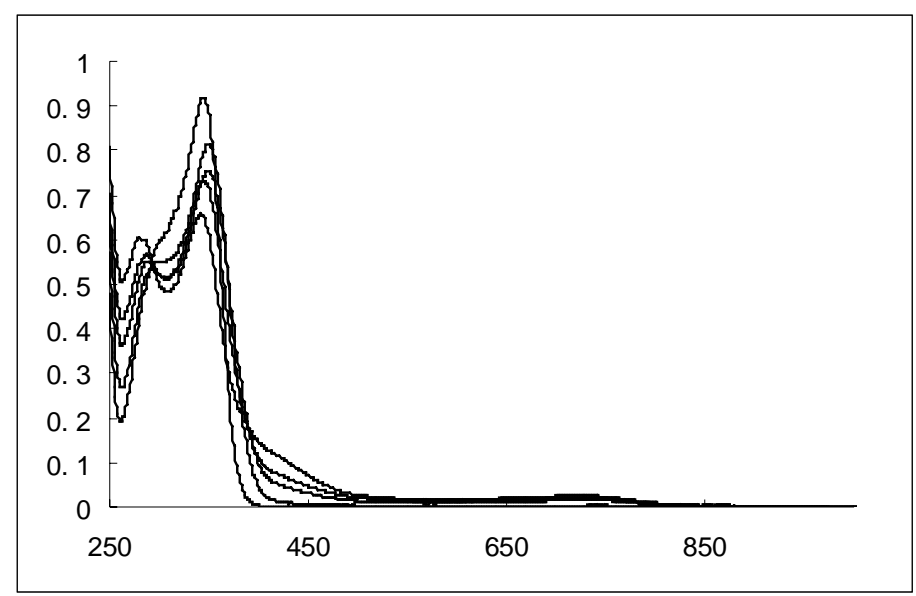

Figure S8. UV-Visible-NIR spectra of polymer 9 b. $\quad\left(3.0 \times 10^{-5} \mathrm{M}\right)$ with $\mathrm{NOBF}_{4}$ (1.0 equiv per aliquot). (Top) Addition of 0.0 to 4.0 equiv. of $\mathrm{NOBF}_{4}$. 

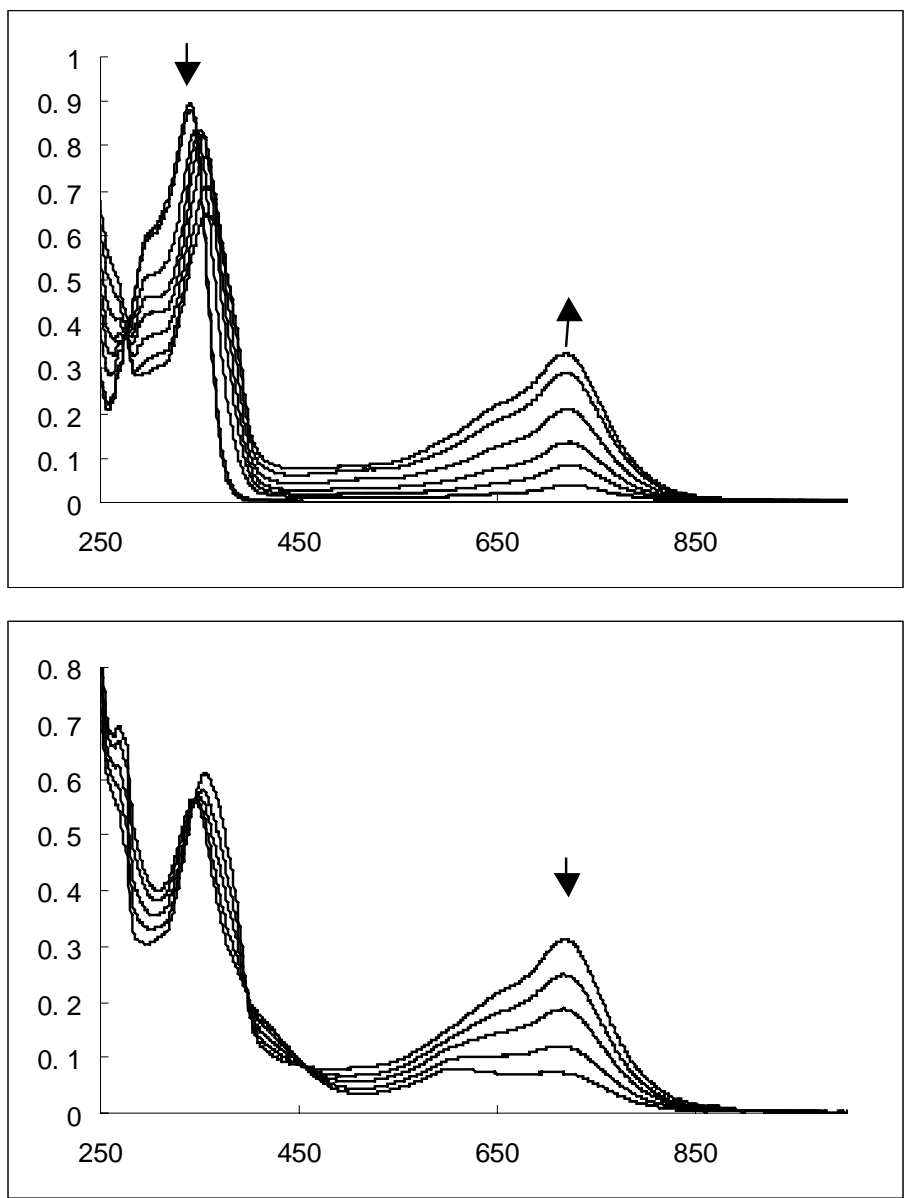

Figure S9. UV-Visible-NIR spectra of Model compound 10b. $\left(2.4 \times 10^{-5} \mathrm{M}\right)$ with $\mathrm{NOBF}_{4}$ (1.0 equiv per aliquot). (Top) Addition of 0.0 to 6.0 equiv. of $\mathrm{NOBF}_{4}$. (Bottom) Addition of 7.0 to 11 equiv. of $\mathrm{NOBF}_{4}$. 

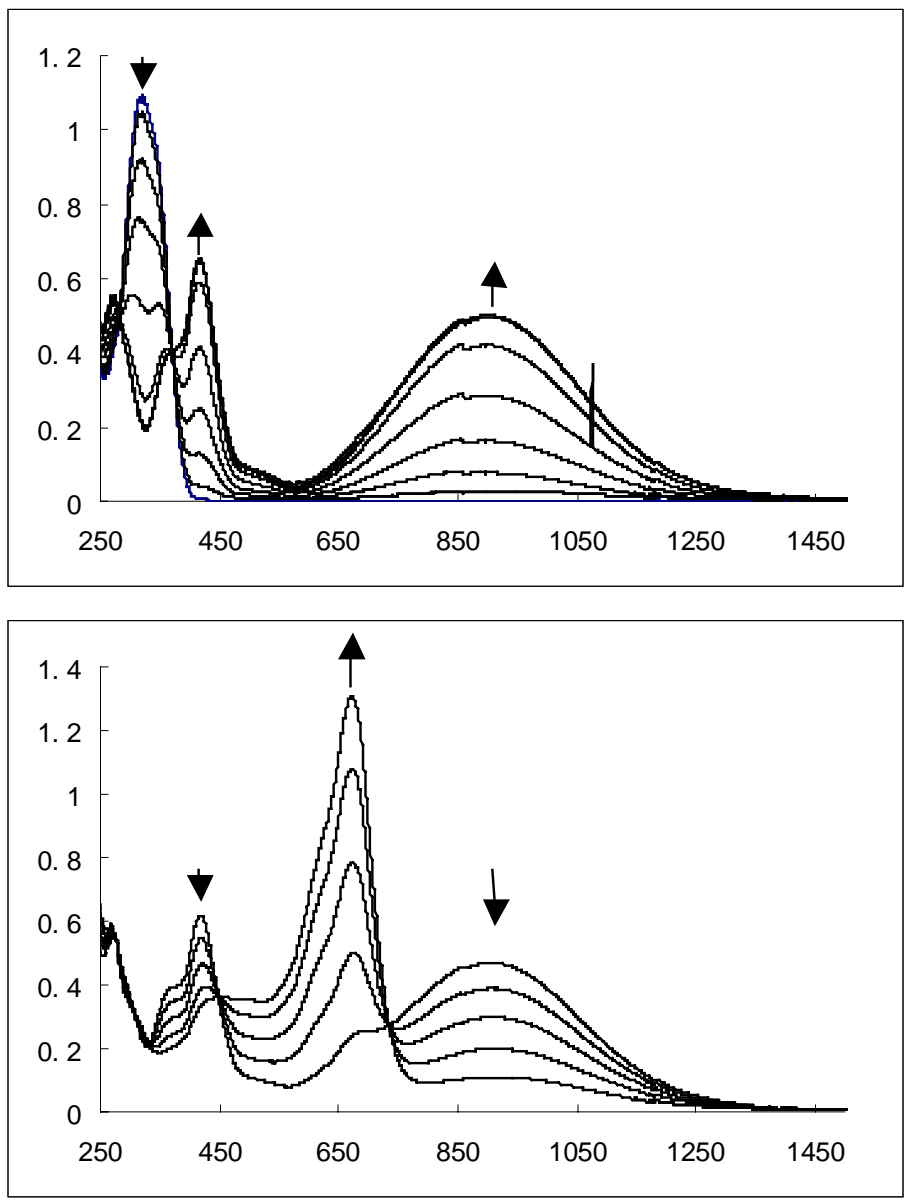

Figure S10. UV-Visible-NIR spectra of polymer 11b. $\left(1.7 \times 10^{-5}\right.$ M) with $\mathrm{NOBF}_{4}$ (0.5 equiv per aliquot). (Top) Addition of 0.0 to 3.0 equiv. of $\mathrm{NOBF}_{4}$. (Bottom) Addition of 3.0 to 5.0 equiv. of $\mathrm{NOBF}_{4}$. 


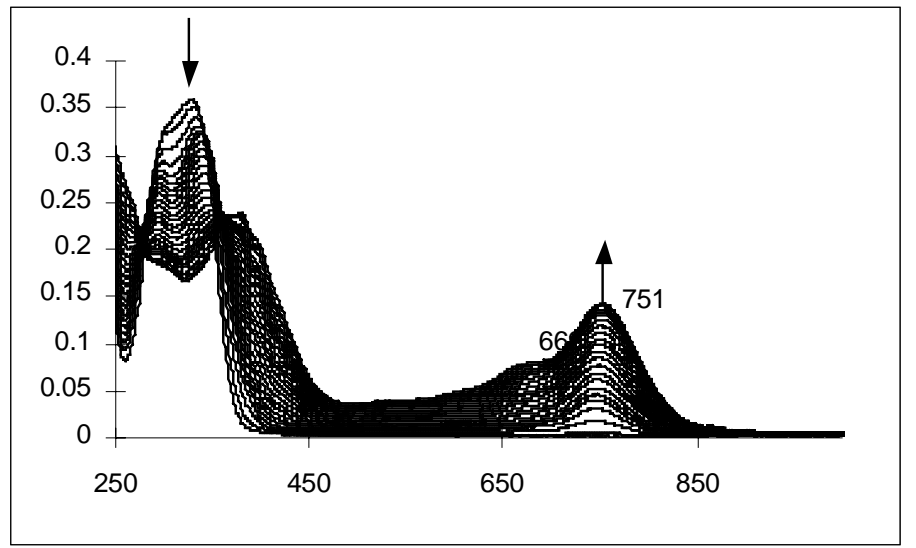

Figure S11. UV-Visible-NIR spectra of polymer 12b. $\quad\left(1.0 \times 10^{-5}\right.$ M) with $\mathrm{NOBF}_{4}$ (0.5 equiv per aliquot). Addition of 0.0 to 13.0 equiv. of $\mathrm{NOBF}_{4}$. 


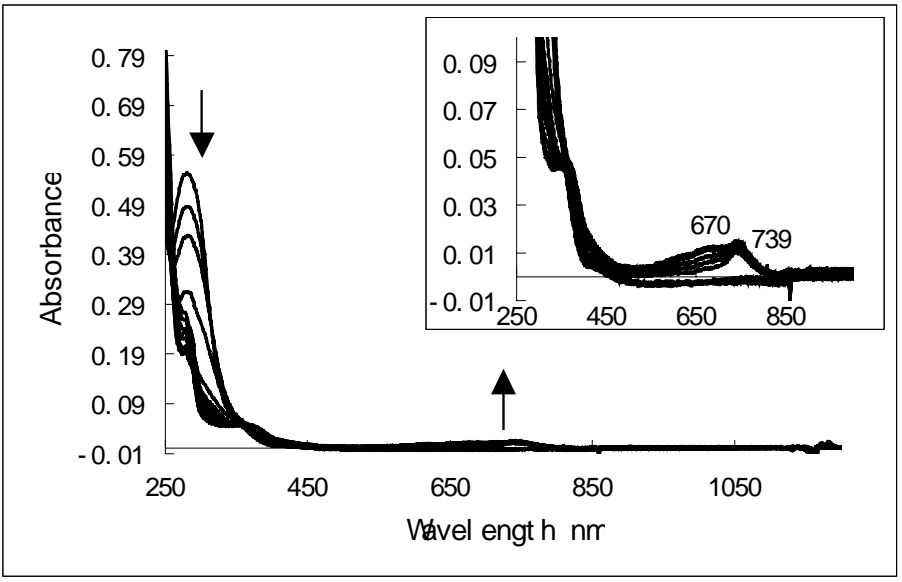

Figure S12. UV-Visible-NIR spectra of polymer 13a. $\quad\left(1.0 \times 10^{-5}\right.$ M) with $\mathrm{NOBF}_{4}$ (0.5 equiv per aliquot). Addition of 0.0 to 8.0 equiv. of $\mathrm{NOBF}_{4}$. 


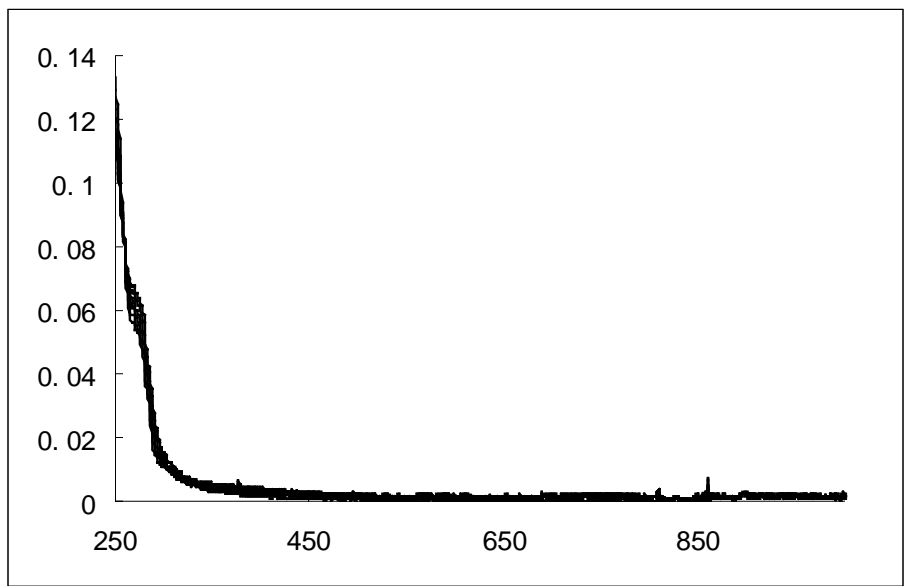

Figure S13. UV-Visible-NIR spectra of polymer 13b. $\quad\left(1.0 \times 10^{-5}\right.$ M) with $\mathrm{NOBF}_{4}$ (1.0 equiv per aliquot). Addition of 0.0 to 5.0 equiv. of $\mathrm{NOBF}_{4}$. 


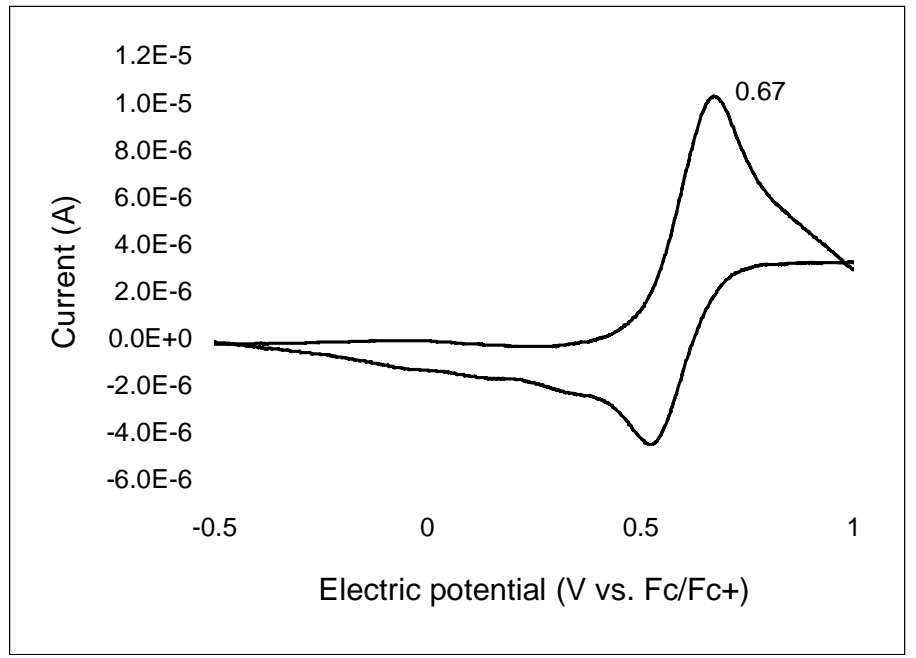

Figure S14. Cyclic Voltammograph for polymers $10 \mathrm{~b}$. 


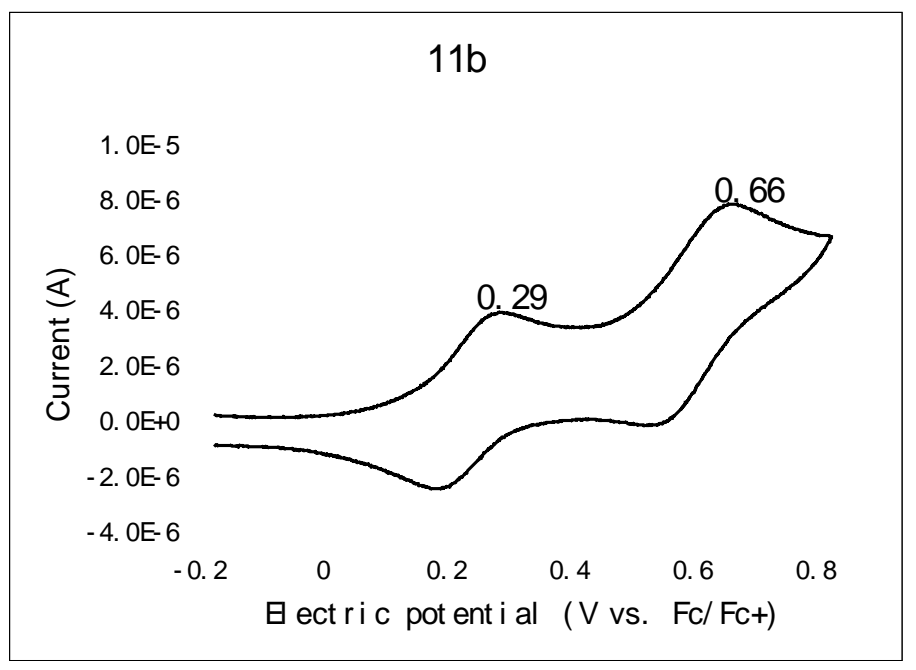

Figure S15. Cyclic Voltammograph for polymers 11b. 


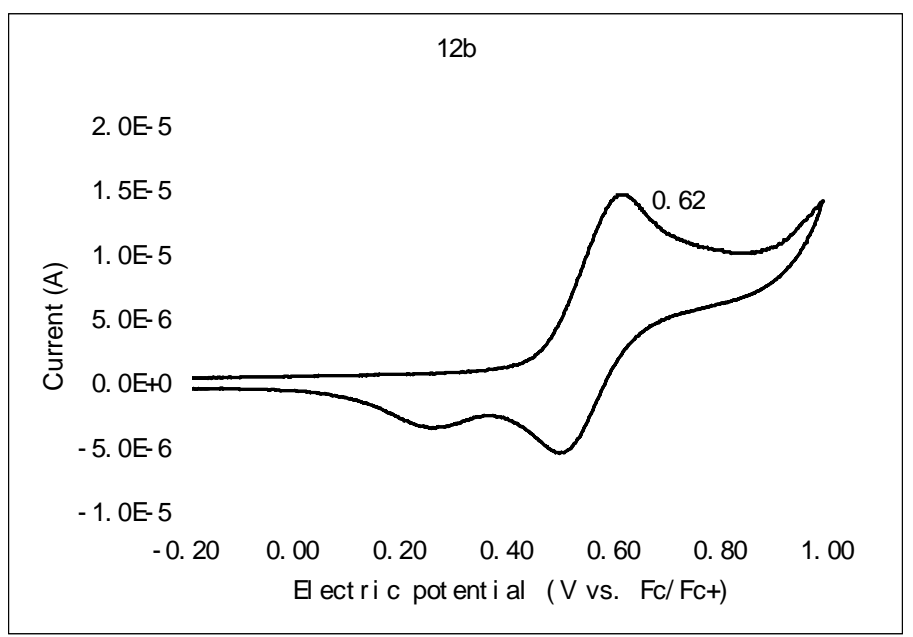

Figure S16. Cyclic Voltammograph for polymer 12b. 